\title{
The dendritic cell receptor DNGR-1 controls endocytic handling of necrotic cell antigens to favor cross-priming of CTLS in virus-infected mice
}

\author{
Santiago Zelenay, ${ }^{1}$ Anna M. Keller, ${ }^{1}$ Paul G. Whitney, ${ }^{1}$ Barbara U. Schraml,, Safia Deddouche, ${ }^{1}$ \\ Neil C. Rogers, ${ }^{1}$ Oliver Schulz, ${ }^{1}$ David Sancho, ${ }^{2}$ and Caetano Reis e Sousa ${ }^{1}$ \\ 1Immunobiology Laboratory, Cancer Research UK, London Research Institute, London, United Kingdom. \\ 2Department of Vascular Biology and Inflammation, CNIC-Centro Nacional de Investigaciones Cardiovasculares, Madrid, Spain.
}

\begin{abstract}
DNGR-1 (CLEC9A) is a receptor for necrotic cells required by DCs to cross-prime CTLs against dead cell antigens in mice. It is currently unknown how DNGR-1 couples dead cell recognition to cross-priming. Here we found that DNGR-1 did not mediate DC activation by dead cells but rather diverted necrotic cell cargo into a recycling endosomal compartment, favoring cross-presentation to $\mathrm{CD8}^{+} \mathrm{T}$ cells. DNGR-1 regulated crosspriming in non-infectious settings such as immunization with antigen-bearing dead cells, as well as in highly immunogenic situations such as infection with herpes simplex virus type 1 . Together, these results suggest that DNGR-1 is a dedicated receptor for cross-presentation of cell-associated antigens. Our work thus underscores the importance of cross-priming in immunity and indicates that antigenicity and adjuvanticity can be decoded by distinct innate immune receptors. The identification of specialized receptors that regulate antigenicity of virus-infected cells reveals determinants of antiviral immunity that might underlie the human response to infection and vaccination.
\end{abstract}

\section{Introduction}

DCs are central players in the establishment and maintenance of self-tolerance, as well as in the induction of immunity against invading microorganisms $(1,2)$. In mice and humans, distinct subsets of DCs can be variably defined by phenotype, ontogeny, and function (3-5). They include the CD8 $\alpha^{+} \mathrm{DC}$ subset found in mouse lymphoid organs and the related CD $103^{+}$DC subset in non-lymphoid tissues, both of which are selectively lost in animals lacking the transcription factor $\operatorname{BATF} 3(6,7)$. Cells bearing a similar phenotype have recently been described in humans, humanized mice, and sheep, indicating cross-species conservation of the CD $8 \alpha^{+}$DC family (8-12). This extended family has distinct functional properties, most notably a remarkable efficiency at capturing material from dead or dying cells, as well as processing exogenous antigens for cross-presentation on $\mathrm{MHC}$ class I (8-11, 13-16). These two features allow CD8 $\alpha^{+}$-like DC to cross-present cell-associated antigens $(17,18)$ and trigger CTL responses against infectious agents or tumors $(6,19-24)$. In addition to priming $\mathrm{CD}^{+} \mathrm{T}$ cells, $\mathrm{CD} 8 \alpha^{+} \mathrm{DCs}$ have been implicated in the establishment of cross-tolerance to tissue-specific cellassociated antigens $(18,25-27)$.

The ability of CD8 $\alpha^{+}$-like DCs to either cross-prime or crosstolerize $\mathrm{CD}^{+} \mathrm{T}$ cells against cell-associated antigens implies that they can decode the context in which they encounter dead cells. In the case of infection, priming may be favored by signals from pattern recognition receptors (PRRs) that recognize bacterial or

Authorship note: Anna M. Keller and Paul G. Whitney contributed equally to this work.

Conflict of interest: The authors have declared that no conflict of interest exists. Citation for this article: J Clin Invest. 2012;122(5):1615-1627. doi:10.1172/JCI60644. viral signatures associated with the dying infected cell $(28,29)$. In the case of uninfected cells such as tumors or allografts, it has been argued that the type of cell death dictates immunogenicity versus tolerogenicity, with necrosis and some forms of apoptosis favoring the former (30-32). This notion implies that CD8 $\alpha^{+}$-like DCs possess receptors that couple innate sensing of necrotic cells to the induction of adaptive immunity. In this regard, we have identified DNGR-1, also known as CLEC9A, as a receptor for necrotic cells that favors cross-priming of CTLs to dead cell-associated antigens in mice (33). DNGR-1 is selectively expressed at high levels by mouse CD8 $\alpha^{+}$DCs $(34,35)$ and $\mathrm{CD}_{103^{+}}$DCs $(36)$ and by their human equivalents $(9,37)$ and recognizes an intracellular ligand that is only exposed after cell death (33). Notably, loss-of-function experiments in the mouse showed that DNGR-1 is not required for dead cell uptake but plays a non-redundant role in priming $\mathrm{CD}^{+} \mathrm{T}$ cells against antigens contained within necrotic cells (33). However, it is currently unknown how DNGR-1 couples dead cell recognition to crosspriming and whether it only controls immunity to cell-associated antigens or is also involved in cross-tolerance.

DNGR-1 is a type II transmembrane C-type lectin receptor (CLR) bearing an extracellular C-type lectin-like domain (CTLD) and a cytoplasmic tail with a hemi-immunoreceptor tyrosine-based activation motif (hemITAM) motif that promotes signaling via spleen tyrosine kinase (Syk) $(34,37)$. The related CLR, Dectin-1, contains a similar hemITAM motif and functions as an activatory receptor in myeloid cells, promoting NF- $\mathrm{\kappa B}$ activation and proinflammatory cytokine production in response to engagement by agonist ligands $(38,39)$. Reflecting the structural similarity between the two receptors, DNGR-1 has been proposed to also act as an activatory CLR (37). Therefore, it is plausible that interaction of DNGR-1 
A

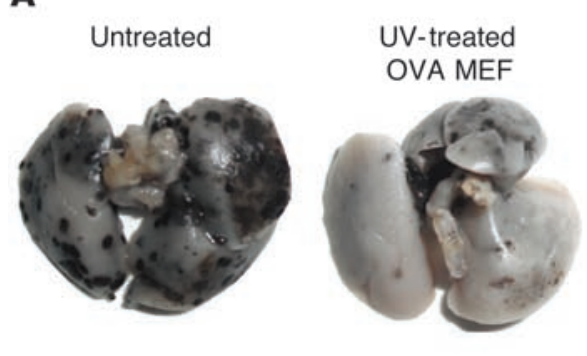

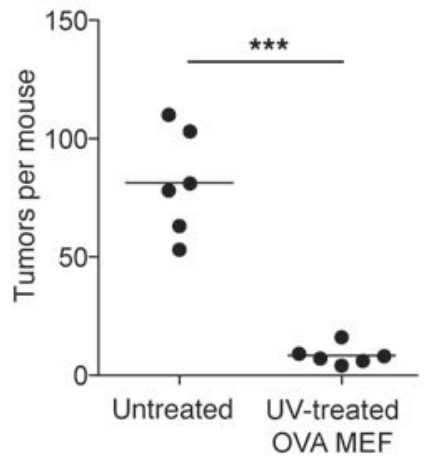

D

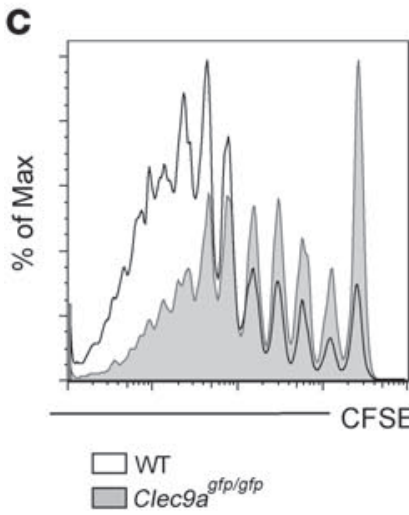

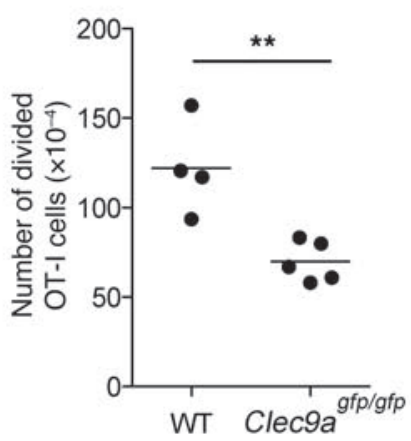

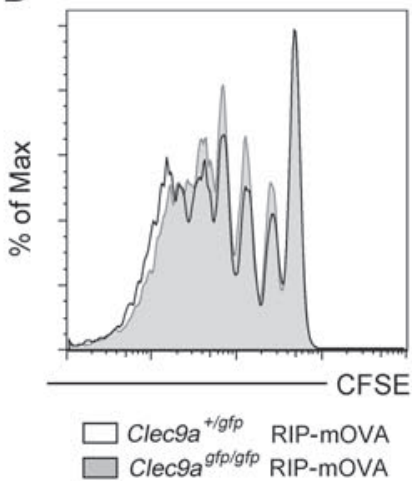

B

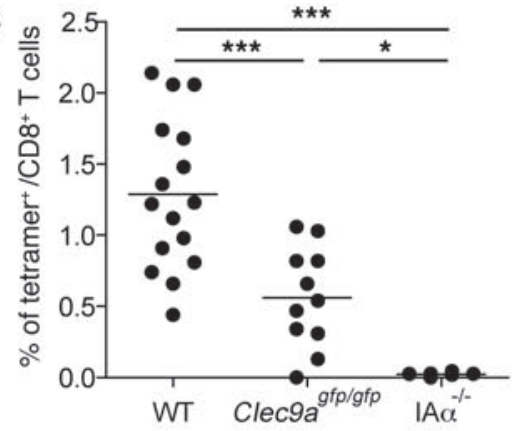

Figure 1

DNGR-1 is required for cross-priming but not for cross-tolerance to dead cell-associated antigens. (A) WT mice were left untreated or were injected with UV-treated $\mathrm{H}-2^{\mathrm{bm} 1}$ OVA-expressing MEFs i.v. One week later mice received B16-OVA cells i.v., and the number of tumors per lung was determined after 18 days. Data are representative of 2 independent experiments ( $n=6 / g r o u p$, each dot represents 1 mouse). (B) WT $(n=16)$, DNGR-1-deficient $(n=11)$, or IA $\alpha^{-/}(n=6)$ mice were injected with UV-treated H-2 ${ }^{\mathrm{bm} 1}$ OVA-expressing MEFs, and the percentage of $\mathrm{H} 2-\mathrm{K}^{\mathrm{b}}-\mathrm{OVA}$ tetramer ${ }^{+}$cells among $\mathrm{CD}^{+} \mathrm{T}$ cells in blood was determined 7 days after injection. Data were pooled from 2 independent experiments; each dot represents 1 mouse. (C) WT or DNGR-1-deficient mice received CFSE-labeled CD45.1+ OT-I cells, followed by immunization with UV-treated OVA-MEFs 1 day later. Spleens were analyzed 5 days after immunization for OT-I proliferation. CFSE dilution (left panel) and total number of divided cells (right panel) are shown; each dot represents 1 mouse. (D) DNGR-1-sufficient or DNGR-1-deficient RIP-mOVA mice received CFSE-labeled CD45.1+ OT-I cells. Renal LNs were analyzed 3 days after injection for OT-I proliferation quantified by CFSE dilution (left panel) and total number of divided cells (right panel); each dot represents 1 mouse. ${ }^{\star} P<0.05$, ${ }^{* \star} P<0.01,{ }^{\star \star \star} P<0.001$, unpaired Student's $t$ test and 1-way ANOVA (Tukey's post-test).

with its ligand on dead cells leads to activation of CD8 $\alpha^{+}$DCs, thereby enhancing their ability to prime naive $\mathrm{CD}^{+} \mathrm{T}$ cells. Consistent with this notion, the function of DNGR-1 in cross-priming to dead cell-associated antigens is dependent on the integrity of the DNGR-1 hemITAM motif and requires Syk kinase (33).

Here, we analyze the role of DNGR-1 in immunity to dead cells and the ability of the receptor to function as a DC activatory receptor. We find that DNGR-1 specifically controls cross-priming, but not cross-tolerance, to cell-associated antigens. However, contrary to expectations, we found that DNGR-1 does not mediate activation of DCs by dead cells. Rather, we show that DNGR-1 has a dedicated function in endocytic handling of necrotic cargo that results in diversion from a degradative fate and favors MHC class I cross-presentation to $\mathrm{CD}^{+} \mathrm{T}$ cells. Consistent with that notion, we show that DNGR-1 deficiency impairs CTL priming even in highly immunogenic situations such as upon infection with herpes simplex virus (HSV) type 1. Thus, DNGR-1 is a dedicated DC receptor for cross-presentation of dead cell-associated antigens that can impact CTL responses to virus infection in mice and may therefore regulate immunity in humans.

\section{Results}

DNGR-1-dependent cross-priming of CTLs against dead cell-associated antigens. The conclusion that DNGR-1 is required for efficient cross-priming of naive $\mathrm{CD}^{+} \mathrm{T}$ cells against antigens borne by dead cells rests on the observation of decreased expansion of antigen-specific $\mathrm{CD}^{+} \mathrm{T}$ cells and impaired development of CTL activity when antigen-bearing dead cells are injected i.v. into DNGR-1-deficient (Clec9asfp/gfp) mice (33). To confirm that this protocol results in immunity, we injected mice with UVtreated secondary necrotic OVA-expressing mouse embryonic fibroblasts (OVA-MEFs) i.v. and then challenged them with an OVA-expressing tumor. Groups that had received the necrotic OVA-MEFs were completely protected from tumor growth, in contrast to untreated mice (Figure 1A). In addition, as crosspriming but not cross-tolerance to dead cell-associated antigens requires $\mathrm{CD}^{+} \mathrm{T}$ cell help $(23,40,41)$, we compared OVA-specific $\mathrm{CD}^{+} \mathrm{T}$ cell expansion in WT versus IA $\alpha^{-/-}$mice after i.v. injection with UV-treated OVA-MEFs. The percentage of OVAspecific $\mathrm{CD}^{+} \mathrm{T}$ cells was severely reduced in $\mathrm{IA}^{-/-}$mice and, in agreement with our previous observations, was also signifi- 
cantly lower in DNGR-1-deficient mice (Figure 1B). Even when precursor frequencies of antigen-specific $\mathrm{CD}^{+} \mathrm{T}$ cells were artificially increased by adoptive transfer of TCR-transgenic CD8 ${ }^{+}$ $\mathrm{T}$ cells (OT-I), i.v. injection of necrotic OVA-MEFs promoted more robust clonal expansion of OVA-specific T cells in WT mice than in DNGR-1-null mice as measured by CFSE dilution and by total cell counts (Figure 1C). Overall, these results confirm and extend our previous observations that immunization with necrotic cells carrying a foreign antigen results in bona fide crosspriming of CTLs, which is partially dependent on DNGR-1.

Cross-tolerance of $\mathrm{CD}^{+} T$ cells against dead cell-associated antigens is DNGR-1 independent. To test whether DNGR-1 might additionally be involved in promoting cross-tolerance to cell-associated antigens, we crossed DNGR-1-deficient mice with RIP-mOVA transgenic mice, which express membrane-bound OVA under the control of the rat insulin promoter (42). In these mice, the crosspresentation of endogenous OVA, presumably from dead or dying cells, promotes proliferation and subsequent deletion of adoptively transferred OT-I T cells $(42,43)$. Notably, and in contrast to mice receiving an inoculum of necrotic cells (Figure 1B), the proliferation of OT-I cells in renal LNs of RIP-mOVA mice was comparable in the DNGR-1-sufficient and -deficient backgrounds, indicating that DNGR-1 is not required for the process culminating in crosstolerization to cell-associated antigens (Figure 1D). Together these results suggest that DNGR-1 plays a specific and non-redundant role in cross-priming, but not in cross-tolerance, against dead cellassociated antigens in vivo.

Activation of $C D 8 \alpha^{+}$DCs by dead cells is DNGR-1 independent. The above results could be interpreted to mean that necrotic cells promote the activation of CD8 $\alpha^{+}$DCs in part by acting through DNGR-1. As a first approach to assess this possibility, we cultured WT and DNGR-1-deficient CD8 $\alpha^{+}$-like DCs (derived from Flt3L cultures of BM cells; ref. 44) with necrotic cells and monitored DC maturation and production of inflammatory cytokines. In comparison to a positive control stimulus, $\mathrm{CpG}$, stimulation with dead cells induced only limited upregulation of co-stimulatory markers (CD86, CD80, CD40) and low levels of secreted IL-12/23p40 but not other proinflammatory cytokines (data not shown and Supplemental Figure 1A; supplemental material available online with this article; doi:10.1172/JCI60644DS1). However, these responses were comparable in cultures of DNGR-1deficient and WT DCs (Supplemental Figure 1A). Additional microarray analysis of the transcriptome of DNGR-1-deficient and WT DCs cultured with dead cells failed to reveal any DNGR-1 dependence in the transcriptional response to the corpses (Supplemental Figure 2). Finally, we injected dead cells into mice and monitored the activation of CD8 $\alpha^{+}$DCs but failed to detect any differences between WT and DNGR-1-deficient animals (Supplemental Figure 1B). Thus, CD $8 \alpha^{+}$DC activation by dead cells appears DNGR-1 independent.

In a separate approach, we carried out experiments with BMderived DCs or macrophages (from GM-CSF or M-CSF BM cultures, respectively) ectopically expressing DNGR-1. These experiments also failed to reveal gain of function at the level of myeloid cell activation upon coculture with dead cells (Supplemental Figure 3). In marked contrast, overexpression of DNGR-1 in Sykexpressing B3Z (B3Z.Syk) cells, a Syk-transduced T cell-derived cell line containing a reporter for NFAT, or in LK cells, a B cellderived cell line, allowed NFAT activation and led to IL-2 and TNF- $\alpha$ production after stimulation with UV-treated dead cells
(Supplemental Figure 4). This response was absent in B3Z.Syk cells expressing a Y7F mutant DNGR-1 (33), indicating that it was hemITAM dependent (Supplemental Figure 4). These results demonstrate that recognition of dead cells by DNGR-1 can induce gene expression and cell activation via hemITAM signaling. However, this response was observed in lymphoid-derived cells ectopically expressing DNGR-1 but absent in myeloid cells constitutively expressing or overexpressing DNGR-1.

DNGR-1 does not act as a myeloid activatory CLR. The inability of DNGR-1 to trigger myeloid cell activation in response to dead cells could result from nullification by simultaneous engagement of inhibitory receptors by the corpses (45). To selectively trigger only DNGR-1, we tried receptor cross-linking with mAbs but failed to detect signs of CD8 $\alpha^{+}$DC activation (Supplemental Figure 5), as reported by others (46). Therefore, we adopted an approach used for human DNGR-1 (37) and generated a chimeric receptor comprising the cytoplasmic tail of mouse DNGR-1 fused to the transmembrane domain and extracellular domain of mouse Dectin-1 (Figure 2A). The chimera can be uniquely triggered using a pure $\beta$-glucan, curdlan (39), thereby circumventing the need for a specific DNGR-1 agonist and obviating the possibility of additional receptor engagement by dead cells.

In order to test for proper folding and signaling capacity of the chimeric receptor, we first expressed it in B3Z.Syk cells. Surface expression of the receptors was monitored with an anti-Dectin-1 $\mathrm{mAb}$ and was comparable in chimera- and Dectin-1-expressing cells (Supplemental Figure 6A). In addition, curdlan stimulation promoted NFAT activation and IL- 2 and TNF- $\alpha$ secretion by B3Z.Syk cells transduced with retroviral vectors encoding either Dectin-1 or the chimeric receptor, but not by cells transduced with an empty vector or a vector encoding a chimeric receptor with a tyrosine to phenylalanine mutation (Y7F) in the hemITAM (Supplemental Figure 6, A and C). Similar results were obtained with LK cells (Supplemental Figure 6B), indicating that the chimeric receptor is able to recognize curdlan and signal for cytokine production with efficiency comparable to that of WT Dectin-1 in lymphoid cells.

We next compared Dectin-1-deficient GM-CSF-grown BMDCs retrovirally transduced to express either Dectin-1 or the chimeric receptor. The retroviral constructs also encoded GFP, which allowed us to sort the transduced cells based on different GFP levels and compare only those with matching levels of surface receptor expression (Figure 2B). Curdlan promoted robust production of TNF- $\alpha$, IL-2, or IL-10 by DCs transduced with Dectin-1-encoding retrovirus but not by DCs transduced with empty control retrovirus encoding only GFP (Figure 2C). In stark contrast, curdlaninduced cytokine production was undetectable or greatly reduced in DCs expressing the chimeric receptor (Figure 2C). A control stimulus, CpG, induced comparable levels of cytokine production by all 3 populations of transduced DCs (Supplemental Figure 7). Similar results were obtained when we used a different chimeric receptor consisting of both the cytoplasmic tail and the transmembrane domain of mouse DNGR-1 fused to the extracellular domain of Dectin-1 (Supplemental Figure 8). These results suggest that, compared with that of Dectin-1, the tail of DNGR-1 is markedly deficient in its ability to signal for cytokine production in myeloid cells.

This conclusion appears to be in contrast to that in a previous report in which RAW264.7 macrophages transduced with an analogous chimeric receptor of human DNGR-1 and mouse 
A
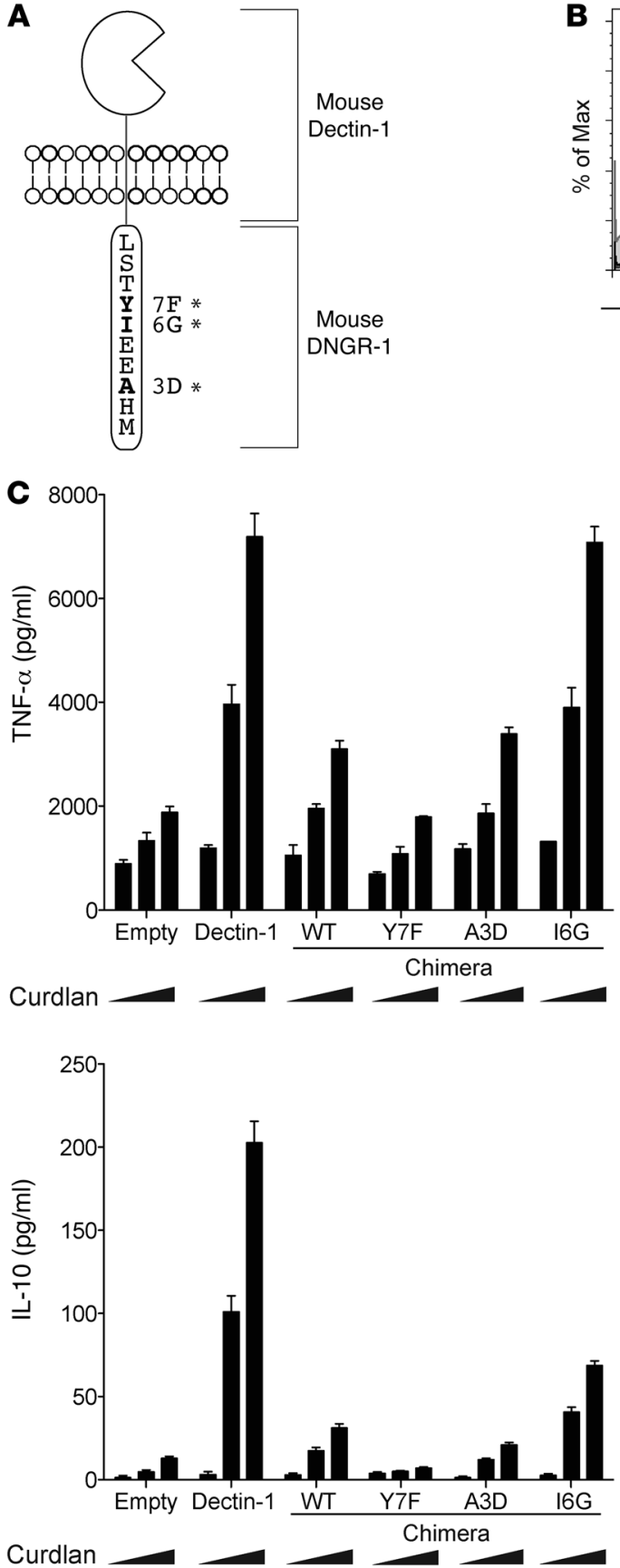
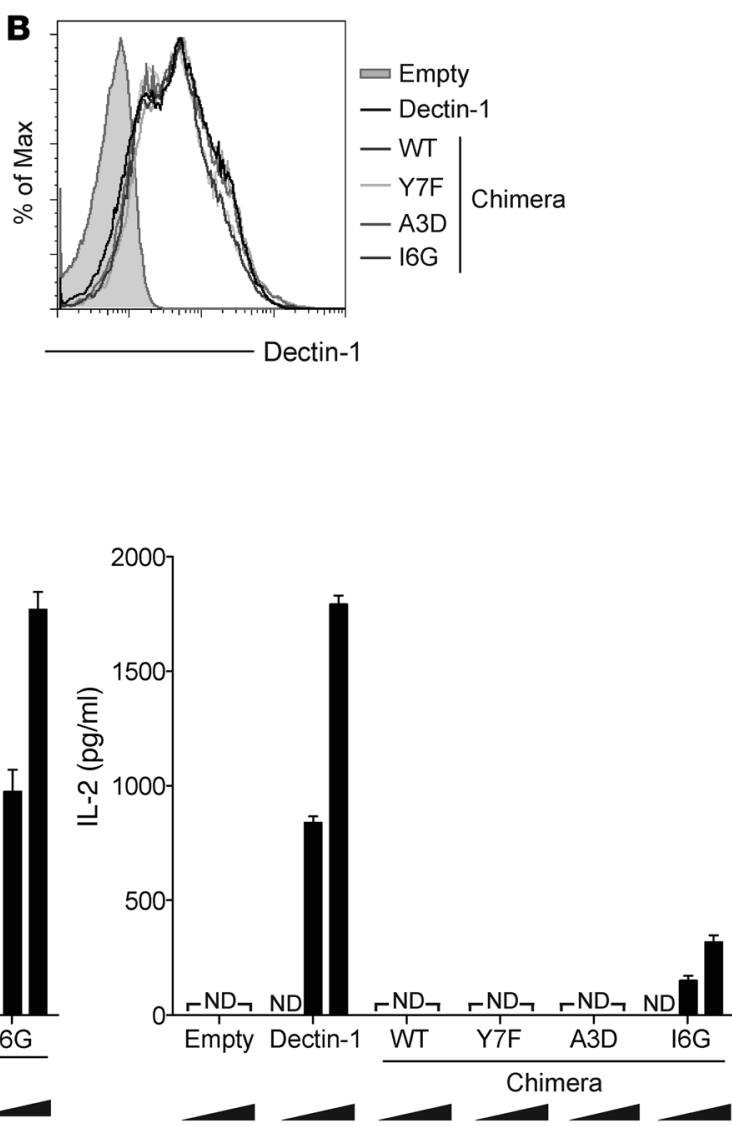

Figure 2

DNGR-1 does not behave as a myeloid activatory receptor. BM cells from Dectin1-deficient mice were retrovirally transduced with a vector encoding for Dectin-1 or for a chimeric receptor (Chimera) bearing the cytoplasmic tail of DNGR-1 (WT) or 3 mutant versions thereof (Y7F, A3D, and I6G) fused to the transmembrane, stalk region, and CTLD of Dectin-1. All constructs were followed by an IRES-GFP sequence, which allowed sorting based on GFP levels to normalize for Dectin-1 or chimera surface expression. (A) Schematic representation of the chimeric DNGR-1/ Dectin-1 receptors. Asterisks denote the mutated residues. (B) Expression of Dectin-1 or the different chimeras by GFP+ GMCSF-BMDCs purified by cell sorting as determined by staining with an anti-Dectin-1 antibody before overnight stimulation. (C) Purified GFP+ DCs as in B were cultured overnight in the presence of increasing amounts of curdlan $(0,30$, and $100 \mu \mathrm{g} / \mathrm{ml})$. Concentrations of TNF- $\alpha$, IL-2, and IL-10 in the supernatant after culture are shown. Results are mean \pm SEM and representative of at least 3 independent experiments. ND, not detected.
Dectin-1 secreted significantly more TNF- $\alpha$ than control cells upon stimulation with zymosan particles (37). Importantly, curdlan specifically triggers Dectin-1, while zymosan can trigger other PRRs such as TLR2 and Dectin-2. It is therefore conceivable that binding of the particles to the chimeric receptor facilitated their recognition by other zymosan-specific receptors, which in turn signaled for TNF- $\alpha$ secretion. To address these possibilities, we procured the cells used in that study (37) and compared their response to zymosan versus curdlan. All cells expressed comparable levels of surface receptor and bound zymosan particles with similar efficiency (Supplemental Figure 9). As reported (37), zymosan induced increased TNF- $\alpha$ produc- tion in cells overexpressing either the chimera or Dectin-1 when compared with control cells transduced with an empty vector (Supplemental Figure 9). However, curdlan led to enhanced TNF- $\alpha$ production only in Dectin-1-transduced cells and not in cells expressing the chimera (Supplemental Figure 9). These results are concordant with our earlier conclusion that signaling by the cytoplasmic tail of DNGR-1 by itself is markedly inefficient at promoting activation of myeloid cells.

Examination of the data revealed a very low level of curdlaninduced cytokines in DCs expressing the Dectin-1/DNGR-1 chimera but not in cells transduced with empty vector (Figure $2 \mathrm{C}$ and Supplemental Figure 8). This appeared to depend on weak signal- 

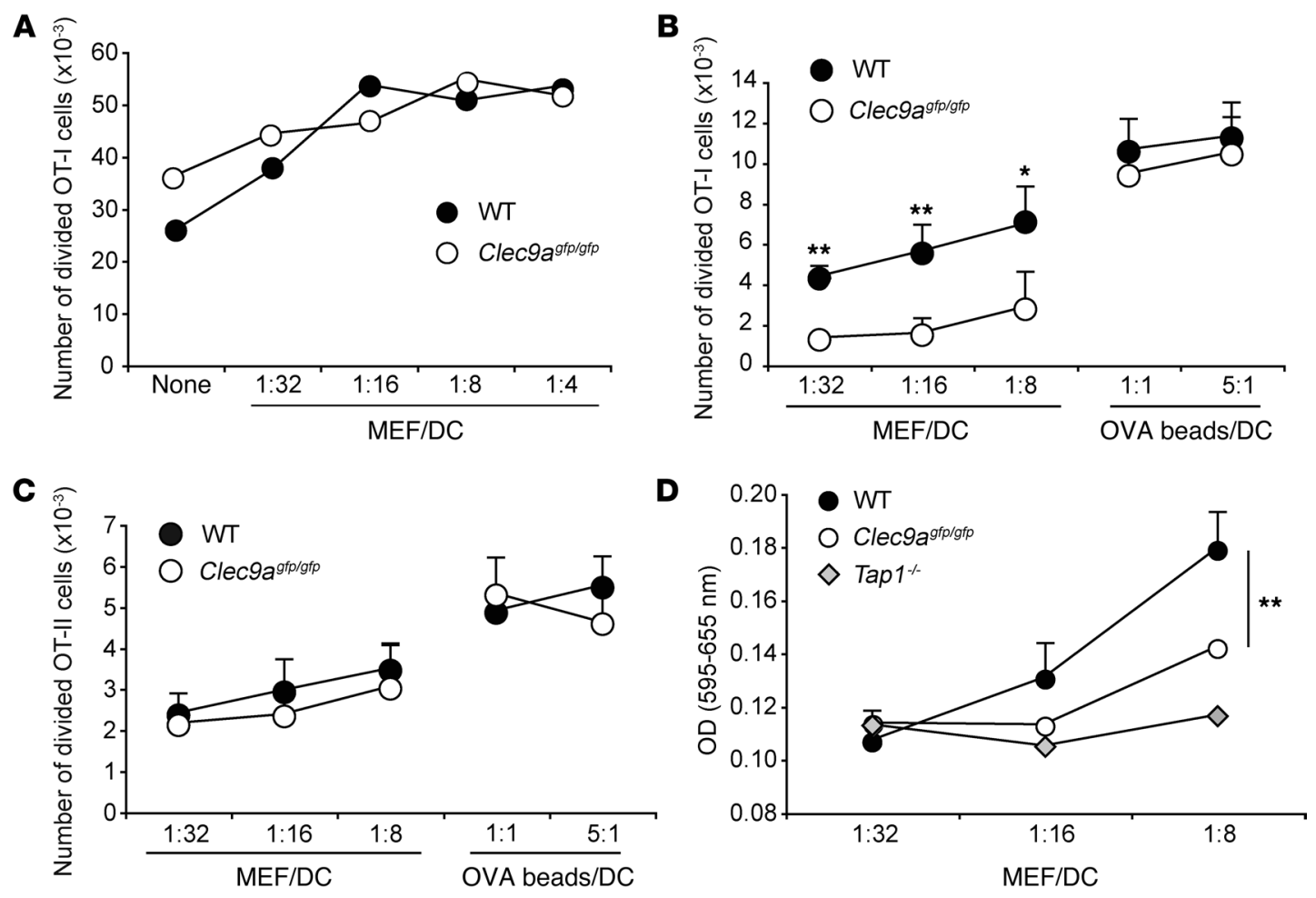

\section{Figure 3}

DNGR-1 regulates MHC class I cross-presentation but not MHC class II presentation of dead cell-associated antigens. (A) The adjuvanticity of dead cells is independent of DNGR-1. Purified CD8 $\alpha^{+}$-like Flt3L-BMDCs from WT or DNGR-1-deficient (Clec9agfp/gfp $)$mice were pulsed with $1 \mathrm{pM}$ SIINFEKL peptide, washed, and cultured with UV-treated $\mathrm{H}-2^{\mathrm{bm} 1} \mathrm{MEFs}$ and CFSE-labeled OT-I T cells. OT-I proliferation was quantified after 3 days. (B and $\mathbf{C}$ ) DNGR-1 regulates cross-presentation of dead cell-associated antigens. Purified CD8 $\alpha^{+}-$like Flt3L-BMDCs from WT or DNGR-1-deficient mice were cultured with UV-treated OVA-MEFs or latex beads coated with OVA (OVA beads). Proliferation of CFSE-labeled OT-I (B) or OT-II (C) T cells was quantified on day 3 or 5 (respectively) of coculture with DCs. (D) Purified CD8 $\alpha^{+}-$like Flt3LBMDCs from WT, DNGR-1-deficient, or Tap1-/- mice were cultured with UV-treated OVA-MEFs and B3Z cells. NFAT reporter activity in B3Z cells was measured using a colorimetric assay after 24 hours of coculture. Results are mean \pm SD and representative of at least 3 independent experiments. ${ }^{*} P<0.05,{ }^{* *} P<0.01$, unpaired Student's $t$ test.

ing by the receptor, as it was not seen with the Y7F mutant (Figure 2C), despite equivalent surface expression of all chimeras (Figure 2B). A DEDG sequence immediately upstream of the hemITAM tyrosine and shared by Dectin-1 and CLEC- 2 but not by DNGR1 has been shown to contribute to signaling by CLEC-2 (47). In DNGR-1 from all species, the glycine was replaced by an isoleucine and, in mouse but not human DNGR-1, it was preceded by two rather than three acidic residues (Figure 2A). We mutated these residues in the Dectin-1/DNGR-1 chimeras to determine whether restoration of a Dectin-1-like DEDG sequence could potentiate the weak signaling by DNGR-1. All chimeras were expressed at similar levels in Dectin-1-deficient BMDCs and signaled to NFAT with comparable efficiency when expressed in B3Z.Syk cells (Figure 2B and Supplemental Figure 6C). However, restoration of the tri-acidic motif had no effect on DC activation by curdlan (Figure 2C). In contrast, the I6G mutant chimeras were able to signal for DC activation as measured by production of TNF- $\alpha$ or IL-2 in response to curdlan, albeit less efficiently than WT Dectin-1 (Figure 2C). We conclude that the DNGR-1 hemITAM can potentially signal to induce myeloid cell activation but this ability is constrained by the presence of an isoleucine residue immediately upstream of the tyrosine.
DNGR-1 deficiency does not impact the adjuvanticity of dead cells. Despite the failure to lead to overt DC activation, weak DNGR-1 signaling could still mediate a subtle change in CD $8 \alpha^{+}$DCs that would lead to an improved ability to stimulate T cells in response to dead cells and account for the role of the receptor in cross-priming. To test for such an effect, we pulsed CD8 $\alpha^{+}$-like Flt3L-derived BMDCs with a limiting concentration of OVA peptide (SIINFEKL) and subsequently allowed them to interact with necrotic MEFs (lacking OVA) before adding naive CFSE-labeled OT-I cells and measuring T cell proliferation. Notably, the expansion of OT-I cells was clearly improved as a function of the ratio of dead cells to DCs (Figure 3A), confirming that dead cell-derived signals improve the stimulatory ability of $\mathrm{CD} 8 \alpha^{+} \mathrm{DCs}$ for naive CD $8^{+} \mathrm{T}$ cells. However, this "adjuvant" effect of dead cells was independent of DNGR-1, as it was seen equally with WT and DNGR-1-deficient DCs (Figure 3A). These results suggest that DNGR-1 does not play a role in DC activation by dead cells even when the latter is measured with a T cell assay that presumably integrates known and unknown parameters of DC activation.

DNGR-1 regulates cross-presentation of dead cell-associated antigens. To reconcile the lack of DC activatory potential with the demonstrable effect of DNGR-1 on cross-priming to dead cell-derived antigens, we hypothesized that DNGR-1 might instead regulate 


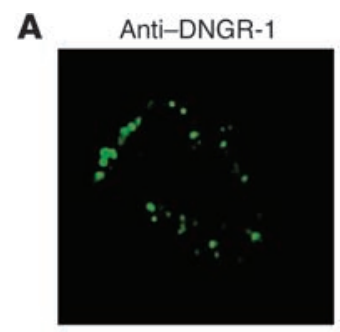

Anti-DNGR-1

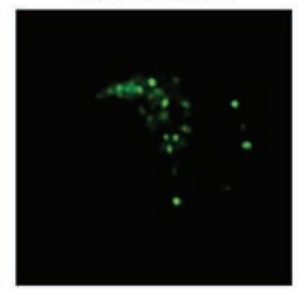

Anti-DNGR-1

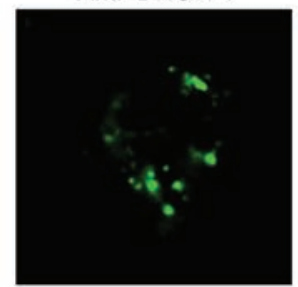

B
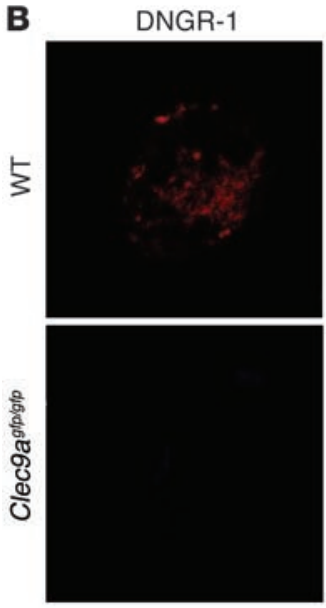

C

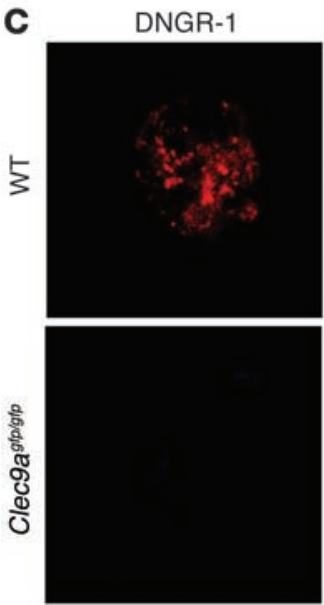

EEA-1

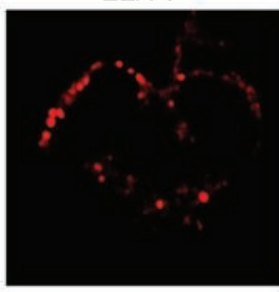

Rab5a

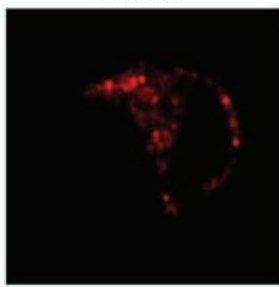

Rab27a
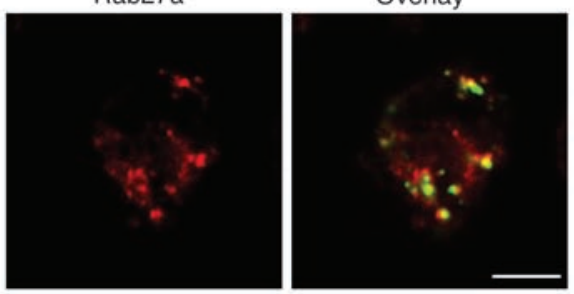

Overlay

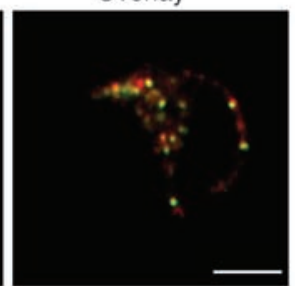

Overlay

MEF

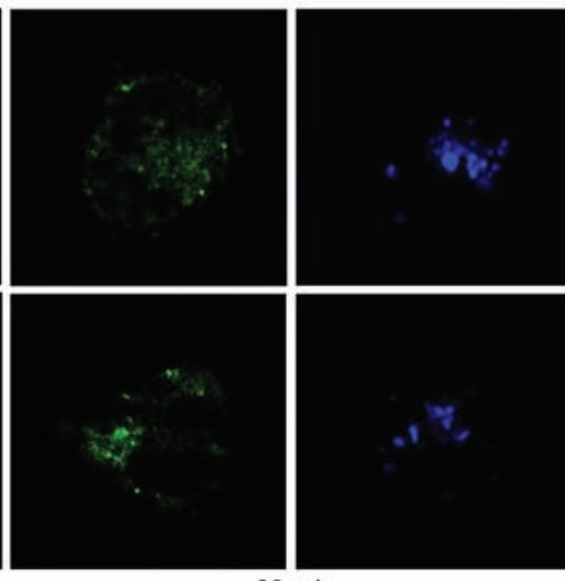

$60 \min$

Rab11

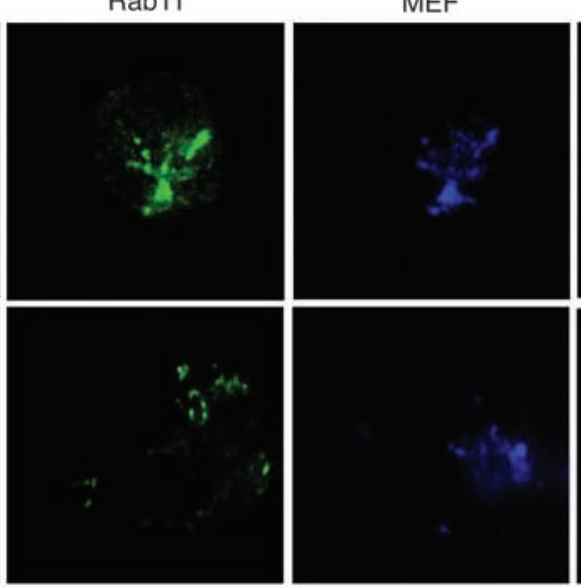

$240 \min$

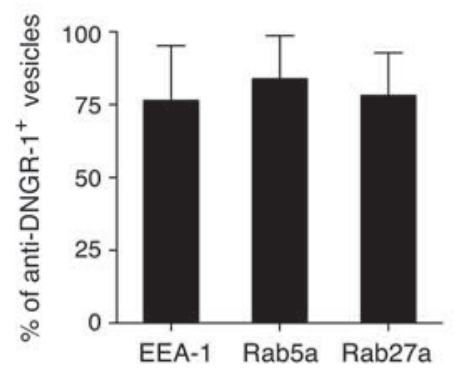

Overlay

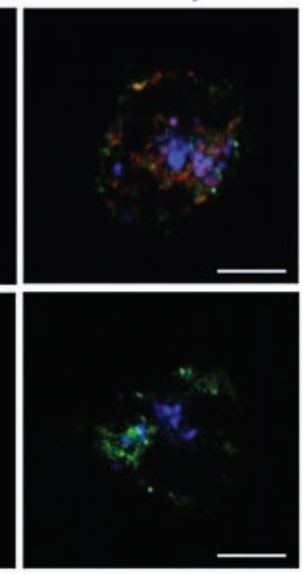

- WT

$\square$ Clec9a gspotp

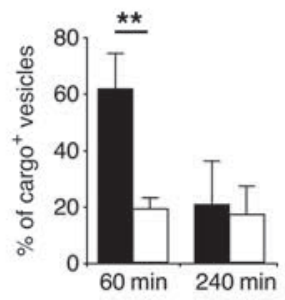

घTT

$\square$ Clec9a gtogtp

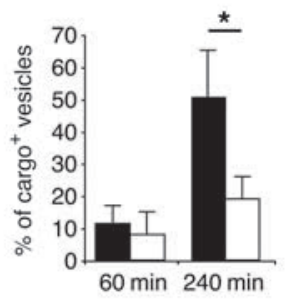




\section{Figure 4}

DNGR-1 diverts cargo to the early/recycling endosomal pool. (A) Purified splenic CD8 $\alpha^{+}$DCs from WT mice were cultured for 30 minutes (EEA-1) or for 90 minutes (Rab5a and Rab27a) with Alexa Fluor 488-coupled anti-DNGR-1 Ab. Cells were fixed and stained for confocal microscopy. Distribution of internalized anti-DNGR-1 $\mathrm{Ab}^{+}$vesicles relative to EEA $-1^{+}, \mathrm{Rab} \mathrm{a}^{+}$, or $\mathrm{Rab} 27 \mathrm{a}^{+}$vesicles was quantified as percentage of vesicles positive for anti-DNGR-1 antibody also positive for endosomal markers in 15 cells per condition. (B and C) Purified CD8 $\alpha^{+}$-like Flt3L-BMDCs from WT or DNGR-1-deficient mice were cultured with UV-treated Alexa Fluor 647-labeled H-2 ${ }^{\mathrm{bm} 1}$ MEFs. Cells were fixed after 60 or 240 minutes and stained for confocal microscopy. Distribution of cargo derived from dead cells relative to Rab5a (B) and Rab11 (C) was quantified as percentage of total vesicles positive for endosomal markers containing dead cell cargo in 15 cells per condition. Scale bars: $5 \mu \mathrm{m}$. Results are representative of 2 independent experiments. ${ }^{\star} P<0.05,{ }^{* *} P<0.01$, unpaired Student's $t$ test.

antigen cross-presentation. We therefore assessed the ability of DNGR-1-deficient CD $8 \alpha^{+}$DCs to stimulate antigen-specific $\mathrm{CD}^{+}$and $\mathrm{CD}^{+} \mathrm{T}$ cells in vitro when antigen was offered in different forms. As reported (33), DNGR-1-deficient DCs were impaired in their ability to support proliferation and clonal expansion of OT-I CD8 ${ }^{+} \mathrm{T}$ cells when antigen was given in the form of necrotic OVA-MEFs but not when it was offered as a conjugate with latex beads (Figure $3 \mathrm{~B}$ ). In contrast, $\mathrm{I}-\mathrm{A}^{\mathrm{b}}$-restricted OVA $_{323-339^{-} \text {-specific OT-II CD4 }}{ }^{+} \mathrm{T}$ cells proliferated and expanded equally after priming by WT and DNGR-1-deficient DCs (Figure 3C). Thus, DNGR-1 deficiency appears to affect the ability of $\mathrm{CD} 8 \alpha^{+} \mathrm{DCs}$ to prime CD8 ${ }^{+} \mathrm{T}$ cells but not $\mathrm{CD} 4^{+} \mathrm{T}$ cells in vitro, consistent with the hypothesis that DNGR-1 controls cross-presentation of dead cell-associated antigens on MHC class I molecules rather than DC activation by cell corpses. Direct assessment of SIINFEKL:H-2 $\mathrm{K}^{\mathrm{b}}$ complexes by mAb staining (48) was not feasible because of sensitivity issues (data not shown), and therefore we measured presentation indirectly by using MHC class I $\left(\mathrm{H}-2^{\mathrm{b}}\right)$-restricted $\mathrm{OVA}_{257-264^{-}}$specific B3Z cells, which respond to antigen without requiring co-stimulation (49). B3Z cells were cocultured with CD $8 \alpha^{+}$-like DCs presenting antigens derived from dead cells, and NFAT reporter activity was assessed after 24 hours (Figure 3D). NFAT reporter activity was lower in B3Z cells cultured with DNGR-1-deficient DCs than those cultured with WT DCs (Figure 3D). To test the dependence on the transporter associated with antigen processing (TAP), we also included Tap $1^{-/}$ DCs in the analysis. The decreased reporter activity in B3Z cells cultured with these DCs confirmed the involvement of TAP during the generation of dead cell-derived peptides for MHC class I loading (Figure 3D). Therefore, DNGR-1 appears to be necessary for efficient cross-presentation of dead cell-associated antigens in a processing pathway that is largely TAP dependent.

DNGR-1 diverts phagocytosed dead cell cargo to a non-degradative recycling endosome compartment. To explain the above results, we tested whether DNGR-1 specifically functions by diverting necrotic cargo to a subcellular compartment that favors cross-presentation, as suggested for another CLR, the mannose receptor (50). We first asked whether DNGR-1 is directed to early endosomal compartments associated with antigen cross-presentation. Consistent with that hypothesis, anti-DNGR-1 mAb bound to the surface of CD $8 \alpha^{+}$ DCs was selectively delivered to vesicles positive for EEA-1, Rab27a, or Rab5a, which mark such compartments $(50,51)$. Indeed, $75 \%$ of DNGR-1 ${ }^{+}$endosomes colocalized with EEA-1 at 30 minutes and with Rab27a or Rab5a at 60-90 minutes after internalization (Figure $4 \mathrm{~A}$ ). We then examined the fate of necrotic cargo taken up by CD $8 \alpha^{+}$-like DCs. We found that DNGR- $1^{+}$vesicles containing dead cell-derived cargo colocalized with Rab5a. Indeed, at 60 minutes of coculture of WT DCs with Alexa Fluor 647-labeled necrotic MEFs, we detected Rab5a in approximately $60 \%$ of vesicles with dead cell-derived material (Figure 4B). This percentage decreased to approximately $20 \%$ at 240 minutes of coculture, indicating that the nature of DNGR- $1^{+}$vesicles changes at later time points after phagocytosis. Interestingly, co-localization of cargo with Rab5a was significantly reduced in DNGR-1-deficient DCs (Figure 4B), suggesting that DNGR-1 may actively determine the intracellular route along which the cargo is directed.

In order to further characterize the nature of the compartment in which cargo associates with DNGR-1, we tested co-localization with Rab11, a marker for recycling endosomes, which have also been implicated in antigen cross-presentation (52). At 60 minutes after uptake, neither WT nor DNGR-1-deficient DCs contained more than $10 \%$ of cargo-positive vesicles that were also Rab $11^{+}$ (Figure 4C). However, at 240 minutes, approximately 50\% of cargocontaining vesicles costained for Rab11 in WT DCs. This co-localization with the recycling endosomal marker was significantly reduced in DCs lacking DNGR-1 (Figure 4C). Together, these data suggest that a proportion of phagocytosed dead cell-derived material associates with DNGR-1 and is diverted to the recycling endosomal route, thereby facilitating cross-presentation.

DNGR-1 is non-redundant for CTL responses to virus infection. If the major role of DNGR-1 were to activate DCs in response to contact with dead cells, its involvement in cross-priming should dissipate when the antigens are derived from virus-infected cells. The latter produce virus nucleic acids that engage PRRs and thereby should overwhelmingly drive DC activation, overriding any putative DNGR-1 contribution. If, on the other hand, the major role of DNGR-1 is to divert cargo to a compartment that facilitates crosspresentation, as argued above, the activity of DNGR-1 should still be apparent even in highly immunogenic conditions such as upon virus infection.

To first test this notion in vitro, we used an OVA-expressing "suicide" cytopathic Semliki Forest virus (SFV) that infects cells and replicates to produce double-stranded RNA but no progeny virions $(28,53)$. Phagocytosis of dead or dying SFV-OVA-infected cells by $C D 8 \alpha^{+}$DCs leads to TLR3-dependent activation of the cells and drives robust cross-priming against OVA (28). Indeed, coculture of SFV-OVA-infected $\mathrm{H}-2^{\mathrm{bm} 1} \mathrm{MEF}$ s with $\mathrm{H}-2^{\mathrm{b}} \mathrm{CD} 8 \alpha^{+}$-like DCs led to marked CD40 and CD86 upregulation, as well as IL- 6 and TNF- $\alpha$ production, all of which were markedly reduced in the absence of TLR3 (Figure 5A). Consistent with this defect in activation and/or an additional effect of TLR3 on cross-presentation, $\mathrm{Tlr}^{-{ }^{--}} \mathrm{CD} 8 \alpha^{+}$ DCs were also impaired in their ability to drive OT-I expansion in response to SFV-OVA-infected cells (Figure 5B). Notably, neither upregulation of DC maturation markers nor the level of cytokines produced was affected by DNGR-1 deficiency (Figure 5A). However, the expansion of OT-I T cells primed by DNGR-1-deficient DCs was still significantly decreased (Figure 5B).

To extend these findings to the context of real virus infection in vivo, we chose a model of cytopathic HSV-1 virus infection of the lung and measured CTL responses in the draining mediastinal LNs by MHC pentamer staining and by restimulating the cells and performing intracellular staining for IFN- $\gamma$ (Supplemental Figure 10). By either measure, CTL responses to the dominant HSV-1 glycoprotein B 

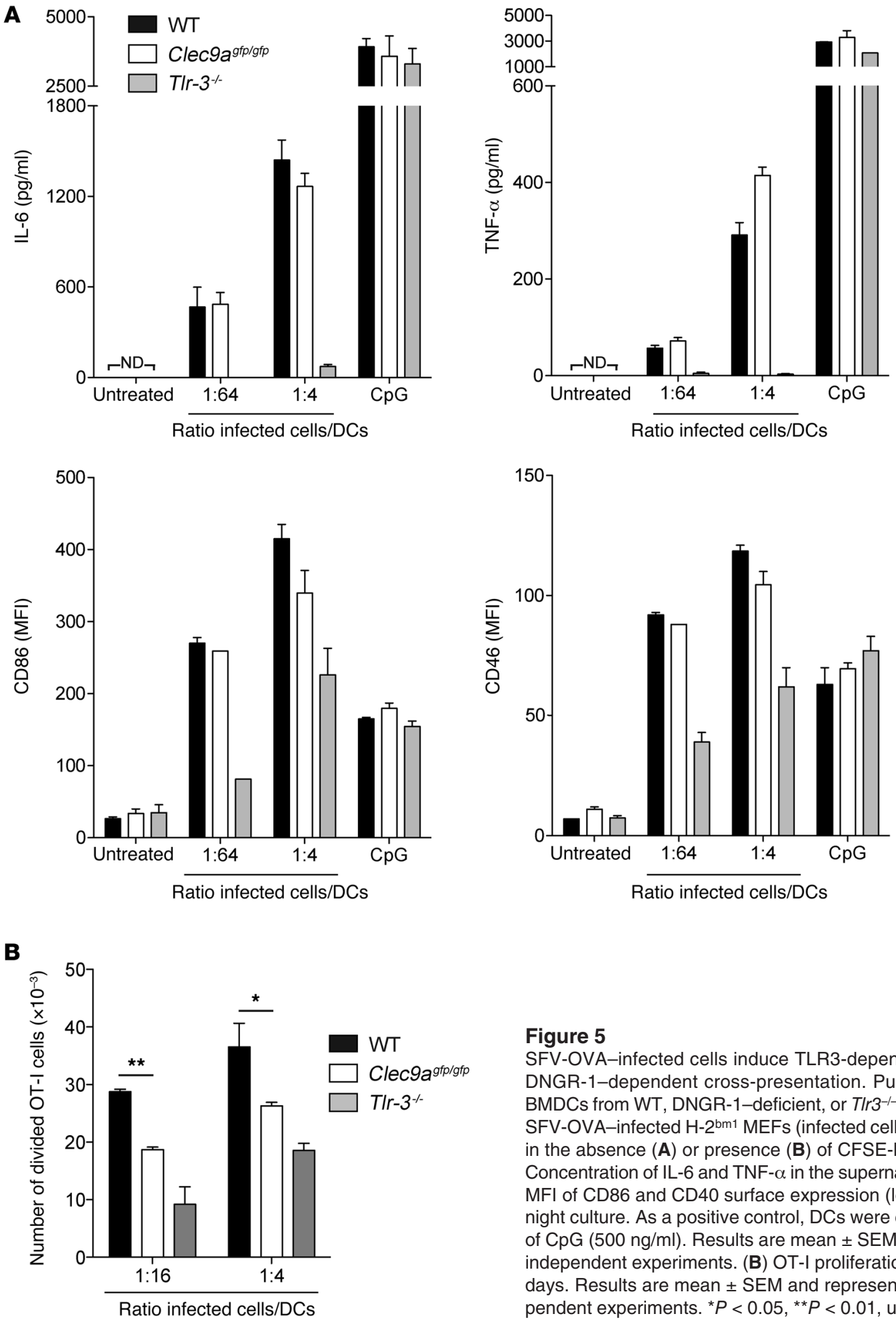

\section{Figure 5}

SFV-OVA-infected cells induce TLR3-dependent DC activation and DNGR-1-dependent cross-presentation. Purified CD8 $\alpha^{+}$-like Flt3LBMDCs from WT, DNGR-1-deficient, or TIr $3^{-/-}$mice were cultured with SFV-OVA-infected $\mathrm{H}-2^{\mathrm{bm} 1} \mathrm{MEFs}$ (infected cells) at the indicated ratios in the absence (A) or presence (B) of CFSE-labeled OT-I T cells. (A) Concentration of IL- 6 and TNF- $\alpha$ in the supernatant (upper panels) and MFI of CD86 and CD40 surface expression (lower panels) after overnight culture. As a positive control, DCs were cultured in the presence of $\mathrm{CpG}(500 \mathrm{ng} / \mathrm{ml})$. Results are mean \pm SEM and representative of 2 independent experiments. (B) OT-I proliferation was quantified after 3 days. Results are mean \pm SEM and representative of at least 3 independent experiments. ${ }^{*} P<0.05,{ }^{* *} P<0.01$, unpaired Student's $t$ test.

(gB) epitope in C57BL/6 mice were markedly reduced in Batf3 $3^{--}$ mice, indicating a major contribution of the CD $8 \alpha^{+}$DC family to $\mathrm{CD}^{+} \mathrm{T}$ cell priming (Figure $6 \mathrm{~A}$ ). Remarkably, a reduction of around $30 \%-80 \%$ in the CTL response to gB was also observed in DNGR-1deficient animals, when measured as number or frequency of gBspecific CTLs (Figure 6A) or by examination of gB peptide-specific

IFN- $\gamma$ production by $\mathrm{CD}^{+} \mathrm{T}$ cells (Figure $6 \mathrm{~B}$ ). In contrast, there was no defect in anti-HSV-1 antibody titers in DNGR-1-deficient mice (Supplemental Figure 11), indicating that DNGR-1 selectively regulates CTL priming. Importantly, no reduction in HSV-1-specific CTL numbers or frequency was seen when DNGR-1-deficient mice were infected via the i.v. route (Supplemental Figure 12). This is 
A

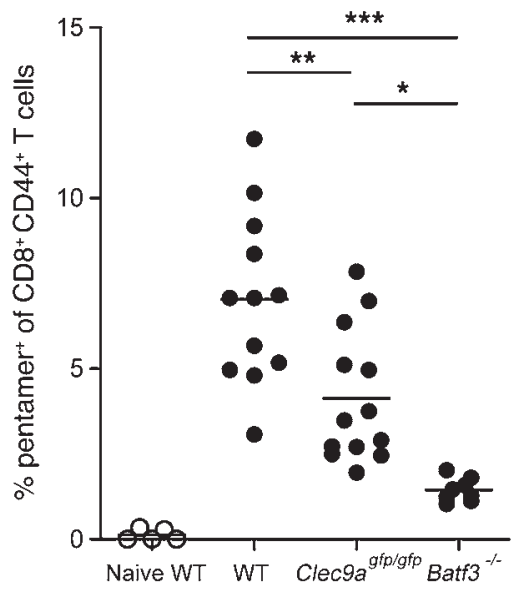

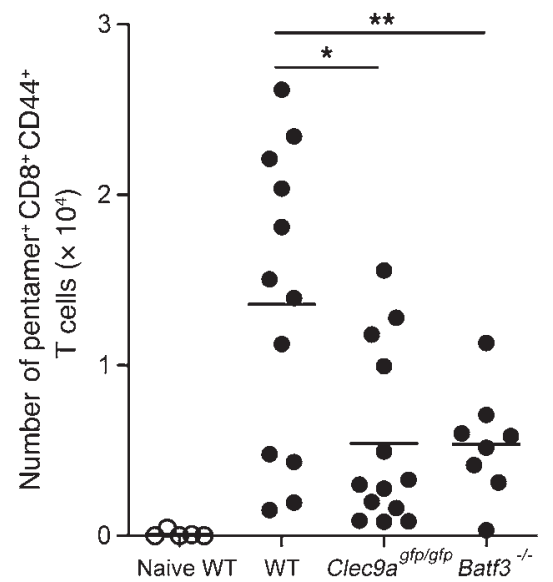

B

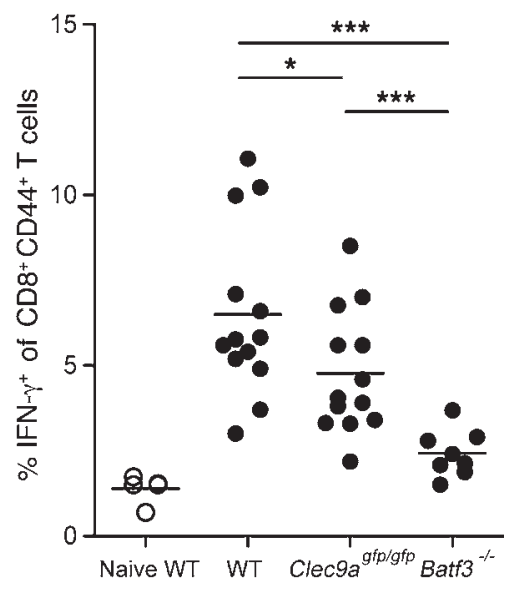

\section{Figure 6}

DNGR-1 contributes to the CTL response to HSV-1 lung infection. WT, DNGR-1-deficient, or Batf3-l- mice were infected with HSV-1 i.n. Mediastinal LNs were harvested 7 days later, and CTL responses were measured directly ex vivo or after in vitro restimulation. (A) Left panel: $\mathrm{H}-2 \mathrm{~K}^{\mathrm{b}}-\mathrm{gB}$ pentamer ${ }^{+}$cells as a percentage of $\mathrm{CD} 8^{+} \mathrm{CD} 44^{+} \mathrm{T}$ lymphocytes. Right panel: Number of $\mathrm{H}-2 \mathrm{~K}^{\mathrm{b}}-\mathrm{gB}$ pentamer ${ }^{+} \mathrm{CD} 8^{+} \mathrm{CD} 44^{+} \mathrm{T}$ cells. (B) IFN- $\gamma^{+}$cells as a percentage of $\mathrm{CD} 8{ }^{+} \mathrm{CD} 44^{+} \mathrm{T}$ lymphocytes after restimulation with gB $\mathrm{B}_{498-505}$ peptide for 6 hours. Data were pooled from 3 independent experiments, with each dot representing 1 mouse; naive WT, $n=5$; WT, $n=13$; DNGR-1-deficient, $n=13$, Batf3---, $n=8$ mice. ${ }^{\star} P<0.05,{ }^{* \star} P<0.01,{ }^{* *} P<0.001,1$-way ANOVA (Tukey's post-test).

likely because cross-priming is dispensable in that setting and establishes that there is no intrinsic defect in the priming of anti-HSV-1 $\mathrm{CD}^{+} \mathrm{T}$ cells in DNGR-1-deficient mice. Thus, DNGR-1 selectively contributes to priming of virus-specific CTLs in a model of lung cytopathic HSV-1 infection, presumably through its ability to regulate cross-presentation of antigens within dying infected cells.

\section{Discussion}

Antigens expressed by dead or dying cells can be made visible to the adaptive immune system, inducing either activation or inactivation of existing lymphocyte repertoires in many experimental models. However, the mechanisms by which innate recognition of cell death is translated into adaptive immunity or tolerance are not fully understood. CD $8 \alpha^{+}$DCs and the related CD $103^{+}$ lineage are thought to be the major DC subsets mediating crosspriming and cross-tolerance to cell-associated antigens $(3,54,55)$. We have shown previously that CD $8 \alpha^{+}$DCs utilize DNGR-1 to recognize a preformed signal exposed in necrotic cells and that ablation or blockade of the receptor reduces their ability to stimulate CTL responses against dead cell-associated antigens in vitro and in vivo (33). Those data and the previously reported function of DNGR-1 as a myeloid activatory CLR (36) suggested that DNGR-1 promotes cross-priming by activating DCs in response to contact with dead cells. Surprisingly, here we failed to find a role for DNGR-1 in promoting DC activation, which is probably regulated by other receptors (32). Rather, we suggest that the receptor acts to regulate the intracellular trafficking of internalized dead cell material, diverting it from a lysosomal proteolytic fate to a non-degradative recycling endosomal compartment that favors antigen extraction for TAP-dependent cross-presentation on MHC class I. We further show that the dedicated function of DNGR-1 in cross-presentation of dead cell-derived antigens contributes in a non-redundant manner to the overall CTL response to HSV-1 lung infection, indicating that it can be a key component of the immune response to cytopathic viruses.
The primacy of the CD $8 \alpha^{+}$DC family in cross-priming to dead cell-associated antigens has been put in question by a recent report suggesting that $\mathrm{CD} 11 \mathrm{c}^{+} \mathrm{CD} 169^{+}$subcapsular sinus macrophages are the primary LN APC cross-presenting necrotic cellderived antigens upon subcutaneous immunization with dead cells (56). Contrary to that report, we found that the CTL response to dead cell-associated antigens remains dependent on DNGR-1 and BATF3 even after subcutaneous immunization (S. Zelenay, B.U. Schraml, and C. Reis e Sousa, unpublished observations). A likely explanation for the discrepancy is that CD169 can be highly expressed by CD8 $\alpha^{+}$DCs and $\mathrm{CD} 103^{+} \mathrm{DCs}$ and can therefore inadvertently serve as a marker for DCs rather than subcapsular sinus macrophages, especially given the high levels of CD11c expressed by the latter (B.U. Schraml, S. Zelenay, and C. Reis e Sousa, unpublished observations). Thus, cross-priming to dead cell-associated antigens remains dependent on DNGR-1 expressed by CD $8 \alpha^{+}$DC family members irrespective of administration route.

Coupling of recognition of dead cells to production of proinflammatory cytokines was previously reported for macrophage-inducible C-type lectin (Mincle) (57). This ITAM-coupled receptor drives production of TNF- $\alpha$ and MIP- 2 and thereby mediates recruitment of neutrophils into the damaged tissue. Mincle and other innate immune receptors, which signal for cellular activation upon recognition of microbial or endogenous ligands, are widely expressed among immune cells. In contrast, DNGR-1 is selectively expressed at high levels by the CD $8 \alpha^{+}$ DC subset, with lower levels of expression on plasmacytoid DCs (34). Moreover, the expression of DNGR-1 on CD8 $\alpha^{+}$DCs is not increased in vivo upon LPS administration in contrast to that of many CLRs with activating functions in myeloid cells (58). Therefore, DNGR-1 exhibits several properties that appear to be unique within the family of C-type lectins that function as innate immune receptors. One additional unique feature underscored in the present study is the apparent separation of cell death sensing from proinflammatory signaling. 
Our conclusion that DNGR-1 does not act by promoting CD $8 \alpha^{+}$ DC activation in response to cell corpses is supported by multiple lines of investigation. First, coculture with dead cells fails to promote DNGR-1-dependent cytokine production or DC maturation. This is the case when testing CD8 $\alpha^{+}$-like FLT3L BMDCs constitutively expressing DNGR-1 or GM-CSF-derived BMDCs and BMderived macrophages ectopically expressing high levels of DNGR-1. Second, using DNGR-1/Dectin-1 receptor chimeras, we found that, unlike Dectin-1, DNGR-1 signaling only weakly induces production of proinflammatory cytokines by DCs. The chimera approach allows us to stimulate cells with a pure Dectin-1 agonist, avoiding the potential co-engagement of multiple agonistic and antagonistic receptors when using dead cells as a stimulus. Notably, the inability of DNGR-1 to signal for cytokine expression can be attributed to specific sequence features of its intracellular tail. Thus, a chimeric receptor consisting of Dectin-1 with the cytoplasmic tail of DNGR-1 was unable to signal for cytokine production, but conversion of a single isoleucine at position 6 into glycine markedly improved stimulatory potential. Third, DC activation triggered by phagocytosis of virus-infected cells was greatly diminished by loss of TLR3 but comparable in WT and DNGR-1-deficient DCs. This finding suggests that, independent of any ability to activate DCs autonomously, DNGR-1 also does not act synergistically with TLR to promote DC activation and further invalidates a model in which DNGR-1 acts by delivering infected cell cargo to TLR3-accessible endosomal compartments. Fourth, loss of DNGR-1 did not affect the ability of CD8 $\alpha^{+}$DCs to stimulate $\mathrm{CD} 4^{+} \mathrm{T}$ cells against dead cell-associated antigens or to stimulate $\mathrm{CD} 8^{+} \mathrm{T}$ cells in response to preprocessed antigen plus necrotic cells as "adjuvants."

Interestingly, DNGR-1 could drive activation in T and B cellderived cell lines, but not in myeloid cells, such as Flt3L BMDCs, GM-CSF BMDCs, BM-derived macrophages, or RAW264.7 macrophages. GM-CSF BMDCs, in particular, are especially amenable to activation by the Dectin-1/Syk pathway, in part because of high expression of CARD9, which permits coupling to NF-кB (59). These data suggest that signaling downstream of Syk-coupled CLR, in particular to NF- $\mathrm{KB}$, is not only cell context dependent (59) but is also controlled in a CLR-specific manner. Further studies will be required to determine the molecular basis of this regulation, which leaves open the possibility that DNGR-1 may still trigger a specific type of CD8 $\alpha^{+}$DC activation in some circumstances that are not mimicked by our present analyses. Nevertheless, collectively, our results do not support the notion that DNGR-1 is a receptor whose primary function is to activate DCs in response to contact with necrotic cells.

Surprisingly, our data highlight an underappreciated aspect of CLR function in regulating endocytic traffic and the retrieval and cross-presentation of dead cell-associated antigens. We have previously demonstrated that DNGR-1+ vesicles in CD $8 \alpha^{+}$DCs do not colocalize with a tracer that accumulates in lysosomes (33). We now find that the receptor and associated dead cell-derived cargo localize to vesicles bearing markers of early endosomes and the recycling endocytic route, indicating that DNGR-1 is confined to the recycling endosome pool. Mannose receptor-mediated uptake and transport of soluble OVA to Rab5a-positive vesicles has been previously reported to favor cross-presentation of OVA-derived peptides (50), and the recycling endosomal compartment is the most favorable point in the endosomal pathway for cross-presentation (60). This may be because recycling endosomes are poorly degradative relative to other endosomal compartments and/or because the recy- cling pathway intersects with a specialized intracellular compartment where acidification is limited by production of NOX2-dependent reactive oxygen species (61). In either case, the limited acidity and proteolytic activity allowed for preservation of intact antigens and translocation into the cytosol for TAP-dependent cross-presentation (61-64). In contrast, generation of peptides for presentation on MHC class II molecules does not require sequestration from degradation, and the presentation of dead cell-associated antigens to $\mathrm{CD}^{+} \mathrm{T}$ cells was not impaired in the absence of DNGR-1. DNGR-1 seems, therefore, to specifically direct dead cell-derived cargo toward a compartment where antigens can be preserved and retrieved for cross-presentation. As such, DNGR-1 may be a useful subcellular marker for identifying and characterizing the elusive "cross-presentation compartment" in CD $8 \alpha^{+}$DCs. Of course, our data do not exclude the possibility that DNGR-1 may additionally control the process of cross-presentation, for example, by promoting fusion with the ER-Golgi intermediate compartment (65) or by facilitating translocation of ligand to the cytosol, as recently shown for another myeloid CLR, the mannose receptor (66). Consistent with that notion, the Y7F hemITAM mutant DNGR-1 still localized to the recycling endosomal compartment even though it was impaired in its ability to promote cross-presentation of dead cell-associated antigens (unpublished observation). Further dissection of the role of DNGR-1 in the regulation of cross-presentation will be greatly facilitated by the identification of the DNGR-1 ligand (67).

Our findings indicate that DNGR-1 regulates cross-priming to dead cell-associated antigens by controlling the antigenicity rather than the adjuvanticity of dead cells. Similarly, it has previously been argued that recognition of HMGB-1 by TLR4 might primarily serve to regulate the ability of DCs to cross-present dead cell antigens (68). If DNGR-1 is involved in regulating cross-presentation rather than DC activation, it might seem odd that its function is apparent only in cross-priming conditions and not in a model of cross-tolerance to cell-associated antigens. This could simply reflect that, during physiological cell death in tissues such as pancreas, the dying cells are cleared before loss of membrane integrity occurs. As the ligands for DNGR-1 are intracellular (33), normal clearance of apoptotic cells during homeostatic conditions may not allow for engagement of the receptor. Even if apoptotic cells are sampled by CD $8 \alpha^{+}$DCs, digestion of the corpse with consequent ligand exposure is likely to occur only in late, degradative endocytic compartments from which, as shown here, DNGR-1 is excluded. Thus, we believe that DNGR-1 involvement is only likely to become apparent in a pathological setting rather than during steady-state physiological cell turnover.

To test for such involvement, we resorted to a model of cytopathic lung infection with HSV-1. Lung contains a prominent population of CD103+ DCs (69) that expresses DNGR-1 at high levels (36) and corresponds to the migratory DCs previously shown to ferry antigen to draining LNs following virus infection $(21,70)$. In addition, DNGR-1-expressing CD $8 \alpha^{+}$DCs in the draining mediastinal LNs can also be prominent APCs for virus-specific $\mathrm{CD}^{+} \mathrm{T}$ cells following lung infection (21). Therefore, the CD $8 \alpha^{+} \mathrm{DC}$ family is likely to be important in inducing CTL responses to respiratory viruses. Consistent with that notion, we found that the CTL response to HSV-1 antigens was markedly reduced in Batf3-deficient mice, which lack $\mathrm{CD} 103^{+}$and $\mathrm{CD} 8 \alpha^{+}$DCs. Importantly, this response was also reduced by an average of 50\% in DNGR-1-deficient mice. HSV-1 lung infection is cleared independently of lymphocytes (71), and therefore, we did not see any increase in virus accumulation in 
the lungs of DNGR-1- or Batf3-deficient mice (data not shown). However, in a different model of vaccinia virus infection, DNGR-1 deficiency clearly impacted virus clearance (72). While we do not know whether the DNGR-1-dependent component reflects crosspriming by the CD8 $\alpha^{+}$DC family or the activity of other cells (e.g., plasmacytoid DCs, which express low levels of the receptor), these findings nevertheless indicate that DNGR-1 can play a key role in the CTL response even under highly immunogenic situations such as virus infection. Our observations therefore suggest a novel and non-redundant point of control in immunity to infection, in which some DC receptors mark dead cells as cargo that contains antigens worthy of cross-presentation, whereas other receptors detect signs of infection in the corpses. As shown here, the former are necessary for cell-associated antigen processing and presentation. In contrast, the latter correspond to typical PRRs, which contribute to effective antigen processing and additionally provide DCs with the activation signals necessary for producing co-stimulatory and other factors that promote effector $\mathrm{T}$ cell development. Manipulation of the pathways involved in dead cell sensing and antigen extraction may have wide implications for the design of vaccines designed to elicit CTLs in infectious disease or cancer immunotherapy. In addition, the identification of specialized receptors that regulate antigenicity of virus-infected cells reveals previously unappreciated determinants of antiviral immunity that might underlie the human response to infection and vaccination.

\section{Methods}

Mice. C57BL/6, Clec9asp/gp (DNGR-1-deficient), Clec7 $7 a^{-1-}$ (Dectin-1-deficient; gift from Gordon Brown, University of Aberdeen, Aberdeen, United Kingdom), Batf $3^{-/}$(gift from Kenneth Murphy, Washington University, St. Louis,

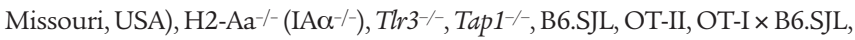
OT-I $\times$ Rag1 $1^{--}$, RIP-mOVA ${ }^{+}$Clec $9 a^{a f p /+}$, and RIP-mOVA ${ }^{+}$Clec $9 a^{a s p / g f p}$ mice were bred at Cancer Research UK in specific pathogen-free conditions.

Cells. Culture medium was RPMI 1640 supplemented with glutamine, penicillin, streptomycin, 2-mercaptoethanol (all from Invitrogen), and 10\% heat-inactivated fetal calf serum (Source BioScience). For T cell cultures, medium was additionally supplemented with non-essential amino acids, HEPES, and sodium pyruvate (Invitrogen). Mouse BMDCs were generated using GM-CSF (GM-CSF-BMDCs) as described previously (73) or by culturing BM cells in the presence of $100-150 \mathrm{ng} / \mathrm{ml} \mathrm{Flt3L}$ (R\&D Systems) for 10 days to generate Flt3L-BMDCs (44). B3Z cells containing a reporter for NFAT coupled to LacZ activity have been previously described (49) and were from N. Shastri (University of California, Berkeley, California, USA). LK35.2 cells (LK cells) (74) were from ATCC (HB-98). OVA-expressing H-2 ${ }^{\mathrm{bm} 1} \mathrm{MEFs}$ (OVA-MEFs) have been described (33). RAW264.7 macrophages stably transduced with an empty retroviral vector or with a retroviral vector coding for mouse Dectin-1 or for a chimeric human DNGR-1/mouse Dectin-1 were previously described (37) and were provided by Gordon Brown. H-2 ${ }^{\mathrm{bm} 1}$ MEFs were infected with OVA-expressing SFV for 2-4 hours as previously described (28). UV-treated OVA-MEFs were generated by exposure to UV light $\left(240 \mathrm{~mJ} / \mathrm{cm}^{2}\right)$, followed by overnight culture in complete medium to induce secondary necrosis, resulting in more than $85 \%$ dead cells. CD $8 \alpha^{+}-$like Flt3LBMDCs were enriched by negative selection using PE-labeled anti-SIRP $\alpha$ and anti-B220 antibodies followed by anti-PE microbeads (Miltenyi Biotec). OT-I T cells were purified from OT-I $\times$ Rag $1^{-/-}$or OT-I $\times$B6.SJL mice and singlecell suspensions of LNs and spleens prepared by liberase/DNAse digestion, followed by negative selection using a cocktail of PE-conjugated antibodies (anti-CD11c, CD11b, B220, MHC II, CD4, NK1.1) and then anti-PE microbeads (Miltenyi Biotec). OT-II cells were similarly purified using an antibody cocktail containing anti-CD8 instead of anti-CD4 mAb.
Injections and infections. Cells were suspended in PBS and injected i.v. into the tail vein or subcutaneously into the flank (200 $\mu$ l per mouse). For immunization with dead cells, mice received $5 \times 10^{5}$ to $7.5 \times 10^{5}$ UV-treated OVA-MEFs. For tumor challenge experiments, mice were untreated or immunized with OVA-expressing UV-treated OVA-MEFs. One week later, mice were given OVA-expressing B16 melanoma cells $\left(2.5 \times 10^{5} /\right.$ mouse $)$ i.v. Mice were sacrificed 18 days after tumor challenge, and tumor burden was assessed by counting lung foci. For virus infection, mice were anesthetized and infected i.n. or i.v. with $1 \times 10^{6} \mathrm{TCID}_{50}$ units of HSV-1 (KOS VR-1493 from ATCC) as previously described (71).

Flow cytometry. Unconjugated anti-Fc $\gamma$ RIII/II, FITC-, PE- or APC-conjugated anti-CD11c, FITC- or PE-coupled anti-CD24, PECy5-coupled and biotinylated anti-CD44, PE-conjugated anti-CD172a (SIRP $\alpha$ ), antiCD11b, anti-NK1.1, anti-CD3, anti-CD45.1, anti-B220, FITC-, PE-, or APC-conjugated anti-CD4, PE-coupled anti-IFN- $\gamma$ and FITC-, PE-, PerCP-, or APC-conjugated anti-CD8 antibodies were all from BD. Alexa Fluor 647-coupled and biotinylated anti-Dectin-1 and isotype-matched control $\mathrm{mAbs}$ were from Serotec. Pacific Blue-coupled anti-CD8 was from BioLegend. Capture anti-IL-2 (BD), anti-IL-10 (BD), anti-IL-12p40 (BD), and anti-TNF- $\alpha(\mathrm{R} \& \mathrm{D})$ and detection biotinylated anti-IL-2 (BD), anti-IL-10 (BD), anti-IL-12p40 (BD), and anti-TNF- $\alpha(\mathrm{R} \& \mathrm{D})$ antibodies were used for ELISA. Mouse IL-10, IL-6, and TNF- $\alpha$ cytometric bead array Flex sets were from BD. Biotinylated anti-DNGR-1 (1F6) was previously described (34). H-2Kb/OVA iTAg MHC tetramers (Beckman Coulter) and $\mathrm{H}-2 \mathrm{~K}^{\mathrm{b}}$ SSIEFARL pentamers (ProImmune) were used for detection of OVA-specific and gB-specific $\mathrm{CD}^{+} \mathrm{T}$ cells, respectively. Cell suspensions from spleen, LNs (prepared by liberase/DNAse digestion), or blood were washed and stained in PBS containing 2\% FCS, 2 mM EDTA, and 0.02\% sodium azide. Cells were incubated with a saturating amount of anti-Fc $\gamma$ RIII/II before staining with the appropriate antibody cocktail. Propidium iodide was added to the final suspension to exclude dead cells before acquisition on a FACSCalibur or Fortessa (BD Biosciences) and analysis performed with FlowJo (Tree Star) software. Live lymphocyte counts were calculated from the acquisition of a fixed number of $10-\mu \mathrm{m}$ latex beads (Beckman Coulter) mixed with a known volume of unstained cell suspension. For intracellular IFN- $\gamma$ staining, cells from mediastinal LNs were restimulated with $1 \mu \mathrm{MgB}_{498-505}$ peptide (synthesized and purified by HPLC at the London Research Institute) for 6 hours. Brefeldin A ( $5 \mu \mathrm{g} / \mathrm{ml}$, Sigma-Aldrich) was added for the last 5 hours of stimulation. Cells were then stained with anti-CD8-Pacifc Blue and anti-CD44-PEcy5, fixed and permeabilized, and then stained with PE-coupled anti-IFN- $\gamma \mathrm{mAb}$.

Chimeric receptors. The complete open reading frame of mouse DNGR-1 or mouse Dectin-1 was cloned upstream of an IRES-GFP sequence into the retroviral vector $\mathrm{pFB}$ and $\mathrm{pMSCV}$. The DNGR-1/Dectin chimeric receptor consisting of the cytoplasmic tail of DNGR-1 fused to the transmembrane, stalk region, and CTLD of Dectin in PFB-IRES-GFP was generated using overlap extension PCR with primers 5'-CCCATTGCAGTGGGTTTAGG-3' and 5'-CCTAAACCCACTGCAATGGGTGCTCCTGAACATTTGCTAG-3'. For the DNGR-1/Dectin chimeric receptor consisting of the cytoplasmic tail and transmembrane region of DNGR-1 fused to the stalk region and CTLD of Dectin in PMSCV, the primers used were $5^{\prime}$-TGCCTTCCTAATTGGATCAT- ${ }^{\prime}$ ' and $5^{\prime}$-ATGATCCAATTAGgAAgGCAGCTGCAGTCACTACCTGAAT-3'. Mutagenesis of DNGR-1 or chimeric receptors was carried out by PCR with primers 5'-TTTCCCGTCGACATGCATGCGGAAGAAGGATATACCTCT-3' for the I6G mutant chimera, 5'-TTTCCCGTCGACATGCATGACGAAGAAATATATACCTCT-3' for the A3D mutant, and 5'-TTTCCCGTCGACATGCATGCGGAAGAAATATTTACCTCT-3' for the Y7F-mutant. All constructs were verified by sequencing. Receptors were introduced into Syk-expressing B3Z cells, LK cells, and Dectin-1-deficient BMDCs by retroviral transduction. 
Cell culture. For DC activation assays in vitro, cells were plated at $2.5 \times$ $10^{5}$ to $5 \times 10^{5} \mathrm{cells} / \mathrm{ml}$ in 96 -well plates at $37^{\circ} \mathrm{C}$ in the presence of different stimuli. After overnight culture, cytokine concentration in the supernatant was determined by ELISA or by cytometric bead array using standard procedures. For cross-priming assays, CD8 $\alpha^{+}$-like Flt3L-BMDCs were cultured at $2.5 \times 10^{5}$ cells $/ \mathrm{ml}$ with purified and CFSE-labeled OVA-specific OT-I or OT-II T cells in 96-well plates for 3 or 5 days, respectively. As a source of antigen, we used UV-treated OVA-MEFs, SFV-OVA-infected MEFs, SIINFEKL peptide (synthesized and purified by HPLC at the London Research Institute), or low-endotoxin OVA protein (Calbiochem) coated on 3- $\mu \mathrm{m}$ polystyrene beads (Sigma-Aldrich). For NFAT reporter assays in B3Z cells, cells were washed in PBS after culture and LacZ activity measured by lysis in CPRG-containing (Roche) buffer. Two to four hours later, OD $595 \mathrm{~nm}$ was measured using OD $655 \mathrm{~nm}$ as a reference.

Confocal laser scanning microscopy. For uptake of anti-DNGR-1 mAb, purified splenic CD8 $\alpha^{+}$DCs from WT mice were cultured in the presence of Alexa Fluor 488-coupled anti-DNGR-1 mAb (7H11, $10 \mu \mathrm{g} / \mathrm{ml})$. For uptake of necrotic MEFs, CD8 $\alpha^{+}$-like DCs from Flt3L-BMDC cultures and Alexa Fluor 647 SE-labeled (Molecular Probes, Invitrogen) UV-treated $\mathrm{H}-2^{\mathrm{bm} 1} \mathrm{MEFs}$ were cocultured in round-bottom 96-well plates. After the indicated periods of time, cells were allowed to adhere for 15 minutes at $37^{\circ} \mathrm{C}$ to coverslips coated with $10 \mu \mathrm{g} / \mathrm{ml}$ fibronectin (Sigma-Aldrich). Cells were then washed in PBS containing $1 \mathrm{mM} \mathrm{MgCl}_{2}$ and $1 \mathrm{mM} \mathrm{CaCl}_{2}$, fixed in $3.7 \%$ paraformaldehyde/PBS for 10 minutes, and permeabilized in $0.1 \%$ Triton X-100/PBS for 3 minutes. After washing with PBS, cells were blocked with PBS containing $1 \%$ bovine serum albumin (SigmaAldrich), $5 \%$ goat serum (Sigma-Aldrich), and anti-Fc $\gamma$ RII/III receptor. DNGR-1 was detected using 7H11 mAb conjugated to Alexa Fluor 546. EEA-1 was detected using a rabbit polyclonal Ab (Santa Cruz Biotechnology Inc.) followed by cross-adsorbed Alexa Fluor 555-labeled goat anti-rabbit IgG antibody (Molecular Probes, Invitrogen). Rab5, Rab11, and Rab27a were detected using mouse mAbs (Synaptic Systems, BD Biosciences, and Santa Cruz Biotechnology Inc., respectively), followed by cross-adsorbed Alexa Fluor 488- or Alexa Fluor 546-labeled goat anti-mouse IgG antibody (Molecular Probes, Invitrogen). Samples were mounted in Fluoromount-G (SouthernBiotech) and analyzed using an Axiovert 100 laser scanning confocal microscope (Zeiss). Image analysis was performed with LSM 510 software (Zeiss) and ImageJ software.

Microarray data. Microarray data have been deposited at the GEO data repository (GEO GSE36435; NCBI tracking system \#16501676).

Statistics. Statistical significance was determined using 1-way ANOVA (Tukey's post-test) and unpaired 2-tailed Student's $t$ test. A $P$ value less than 0.05 was considered significant.

Study approval. All animal experiments were performed in accordance with national and institutional guidelines for animal care and were approved by the London Research Institute Animal Ethics Committee and by the Home Office, UK.

\section{Acknowledgments}

Work in the C. Reis e Sousa laboratory is funded by Cancer Research UK and a prize from Fondation Bettencourt-Schueller. S. Zelenay, A.M. Keller, and B.U. Schraml were supported by EMBO long-term fellowships and by a Marie Curie Intra-European Fellowship to S. Zelenay. P.G. Whitney was supported by an Overseas Biomedical Fellowship from the National Health and Medical Research Council of Australia. D. Sancho is a recipient of a Ramón y Cajal fellowship from the Spanish Ministry of Innovation and Science. We thank Gordon Brown for RAW264.7 macrophages expressing the human DNGR-1/ mouse Dectin-1 chimeric receptor and Philip East for microarray analysis. We are grateful to members of the Cancer Research UK Immunobiology Laboratory for assistance and helpful discussions.

Received for publication August 23, 2011, and accepted in revised form February 29, 2012.

Address correspondence to: Caetano Reis e Sousa, Immunobiology Laboratory, Cancer Research UK, London Research Institute, Lincoln's Inn Fields Laboratories, 44 Lincoln's Inn Fields, London WC2A 3LY, United Kingdom. Phone: 44.20.7269.2832; Fax: 44.20.7269.2833; E-mail: caetano@cancer.org.uk.
1. Steinman RM. Lasker Basic Medical Research Award. Dendritic cells: versatile controllers of the immune system. Nat Med. 2007;13(10):1155-1159.

2. Steinman RM. Dendritic cells in vivo: a key target for a new vaccine science. Immunity. 2008;29(3):319-324.

3. Villadangos JA, Schnorrer P. Intrinsic and cooperative antigen-presenting functions of dendritic-cell subsets in vivo. Nat Rev Immunol. 2007;7(7):543-555.

4. Heath WR, Carbone FR. Dendritic cell subsets in primary and secondary $\mathrm{T}$ cell responses at body surfaces. Nat Immunol. 2009;10(12):1237-1244.

5. Merad M, Manz MG. Dendritic cell homeostasis. Blood. 2009;113(15):3418-3427.

6 . Hildner $\mathrm{K}$, et al. Batf3 deficiency reveals a critical role for CD8alpha+ dendritic cells in cytotoxic $\mathrm{T}$ cell immunity. Science. 2008;322(5904):1097-1100.

7. Edelson BT, et al. Peripheral CD103+ dendritic cells form a unified subset developmentally related to CD8alpha+ conventional dendritic cells. J Exp Med. 2010;207(4):823-836.

8. Jongbloed SL, et al. Human CD141+ (BDCA-3)+ dendritic cells (DCs) represent a unique myeloid DC subset that cross-presents necrotic cell antigens. J Exp Med. 2010;207(6):1247-1260.

9. Poulin LF, et al. Characterization of human DNGR$1+$ BDCA3 + leukocytes as putative equivalents of mouse CD8alpha+ dendritic cells. J Exp Med. 2010;207(6):1261-1271.

10. Bachem A, et al. Superior antigen cross-presentation and XCR1 expression define human CD11c+CD141+ cells as homologues of mouse CD8+ dendritic cells.
JExpMed. 2010;207(6):1273-1281.

11. Crozat $\mathrm{K}$, et al. The XC chemokine receptor 1 is a conserved selective marker of mammalian cells homologous to mouse CD8alpha+ dendritic cells. J Exp Med. 2010;207(6):1283-1292.

12. Contreras V, et al. Existence of CD8alpha-like dendritic cells with a conserved functional specialization and a common molecular signature in distant mammalian species. J Immunol. 2010;185(6):3313-3325.

13. Schulz O, Reis e Sousa C. Cross-presentation of cell-associated antigens by CD8alpha + dendritic cells is attributable to their ability to internalize dead cells. Immunology. 2002;107(2):183-189.

14. Iyoda T, et al. The CD8+ dendritic cell subset selectively endocytoses dying cells in culture and in vivo. J Exp Med. 2002;195(10):1289-1302.

15. Schnorrer $\mathrm{P}$, et al. The dominant role of CD8+ dendritic cells in cross-presentation is not dictated by antigen capture. Proc Natl Acad Sci U S A. 2006;103(28):10729-10734.

16. Dudziak D, et al. Differential antigen processing by dendritic cell subsets in vivo. Science. 2007;315(5808):107-111.

17. den Haan JM, Lehar SM, Bevan MJ. CD8(+) but not CD8(-) dendritic cells cross-prime cytotoxic T cells in vivo. J Exp Med. 2000;192(12):1685-1696.

18. Belz GT, et al. The CD8alpha(+) dendritic cell is responsible for inducing peripheral self-tolerance to tissue-associated antigens. J Exp Med. 2002;196(8):1099-1104.

19. Allan RS, et al. Epidermal viral immunity induced by CD8alpha+ dendritic cells but not by Langerhans cells. Science. 2003;301(5641):1925-1928.

20. Belz GT, et al. Cutting edge: conventional CD8 alpha+ dendritic cells are generally involved in priming CTL immunity to viruses. I Immunol. 2004;172(4):1996-2000.

21. Belz GT, et al. Distinct migrating and nonmigrating dendritic cell populations are involved in MHC class I-restricted antigen presentation after lung infection with virus. Proc Natl Acad Sci U S A. 2004;101(23):8670-8675.

22. Allan RS, et al. Migratory dendritic cells transfer antigen to a lymph node-resident dendritic cell population for efficient CTL priming. Immunity. 2006;25(1):153-162.

23. Bennett SR, Carbone FR, Karamalis F, Miller JF, Heath WR. Induction of a CD8+ cytotoxic T lymphocyte response by cross-priming requires cognate CD4+ T cell help. J Exp Med. 1997;186(1):65-70.

24. Bedoui S, et al. Cross-presentation of viral and self antigens by skin-derived CD103+ dendritic cells. Nat Immunol. 2009;10(5):488-495.

25. Ferguson TA, Herndon J, Elzey B, Griffith TS, Schoenberger S, Green DR. Uptake of apoptotic antigen-coupled cells by lymphoid dendritic cells and cross-priming of CD8(+) T cells produce active immune unresponsiveness. I Immunol. 2002;168(11):5589-5595.

26. Luckashenak N, et al. Constitutive crosspresentation of tissue antigens by dendritic cells controls CD8+ $\mathrm{T}$ cell tolerance in vivo. Immunity. 
2008;28(4):521-532.

27. Qiu CH, Miyake Y, Kaise H, Kitamura H, Ohara O, Tanaka M. Novel subset of CD8\{alpha\}+ dendritic cells localized in the marginal zone is responsible for tolerance to cell-associated antigens. J Immunol. 2009;182(7):4127-4136.

28. Schulz O, et al. Toll-like receptor 3 promotes cross-priming to virus-infected cells. Nature. 2005;433(7028):887-892.

29. Torchinsky MB, Garaude J, Martin AP, Blander JM. Innate immune recognition of infected apoptotic cells directs $\mathrm{T}(\mathrm{H}) 17$ cell differentiation. Nature. 2009;458(7234):78-82.

30. Matzinger P. The danger model: a renewed sense of self. Science. 2002;296(5566):301-305.

31. Kroemer G, Zitvogel L. Death, danger, and immunity: an infernal trio. Immunol Rev. 2007;220:5-7.

32. Green DR, Ferguson T, Zitvogel L, Kroemer G. Immunogenic and tolerogenic cell death. Nat Rev Immunol. 2009;9(5):353-363.

33. Sancho D, et al. Identification of a dendritic cell receptor that couples sensing of necrosis to immunity. Nature. 2009;458(7240):899-903.

34. Sancho D, et al. Tumor therapy in mice via antigen targeting to a novel, DC-restricted C-type lectin. J Clin Invest. 2008;118(6):2098-2110.

35. Caminschi I, et al. The dendritic cell subtyperestricted C-type lectin Clec9A is a target for vaccine enhancement. Blood. 2008;112(8):3264-3273.

36. Poulin LF, et al. DNGR-1 is a specific and universal marker of mouse and human Batf3-dependent dendritic cells in lymphoid and non-lymphoid tissues [published online ahead of print March 22, 2012]. Blood. doi:10.1182/blood-2012-01-406967.

37. Huysamen C, Willment JA, Dennehy KM, Brown GD. CLEC9A is a novel activation C-type lectinlike receptor expressed on BDCA3+ dendritic cells and a subset of monocytes. J Biol Chem. 2008;283(24):16693-16701.

38. Gross $\mathrm{O}$, et al. Card 9 controls a non-TLR signalling pathway for innate anti-fungal immunity. Nature. 2006;442(7103):651-656

39. LeibundGut-Landmann S, et al. Syk- and CARD9dependent coupling of innate immunity to the induction of $\mathrm{T}$ helper cells that produce interleukin 17. Nat Immunol. 2007;8(6):630-638.

40. Kurts C, et al. CD4+ T cell help impairs CD8+ T cell deletion induced by cross-presentation of self-antigens and favors autoimmunity. J Exp Med. 1997;186(12):2057-2062.

41. Griffith TS, et al. Apoptotic cells induce tolerance by generating helpless CD8+ T cells that produce TRAIL. JImmunol. 2007;178(5):2679-2687.

42. Kurts C, Heath WR, Carbone FR, Allison J, Miller JF, Kosaka H. Constitutive class I-restricted exogenous presentation of self antigens in vivo. $J$ Exp Med. 1996;184(3):923-930.

43. Kurts C, Kosaka H, Carbone FR, Miller JF, Heath WR. Class I-restricted cross-presentation of exogenous self-antigens leads to deletion of autoreactive
CD8(+) T cells. J Exp Med. 1997;186(2):239-245.

44. Naik SH, et al. Cutting edge: generation of splenic CD8+ and CD8- dendritic cell equivalents in Fmslike tyrosine kinase 3 ligand bone marrow cultures. J Immunol. 2005;174(11):6592-6597.

45. Chen GY, Tang J, Zheng P, Liu Y. CD24 and Siglec-10 selectively repress tissue damage-induced immune responses. Science. 2009;323(5922):1722-1725.

46. Lahoud MH, et al. Targeting antigen to mouse dendritic cells via Clec9A induces potent CD4 T cell responses biased toward a follicular helper phenotype. J Immunol. 2011;187(2):842-850.

47. Fuller GL, et al. The C-type lectin receptors CLEC-2 and Dectin-1, but not DC-SIGN, signal via a novel YXXL-dependent signaling cascade. J Biol Chem. 2007;282(17):12397-12409

48. Porgador A, Yewdell JW, Deng Y, Bennink JR, Germain $\mathrm{RN}$. Localization, quantitation, and in situ detection of specific peptide-MHC class I complexes using a monoclonal antibody. Immunity. 1997;6(6):715-726.

49. Karttunen J, Sanderson S, Shastri N. Detection of rare antigen-presenting cells by the lacZ T-cell activation assay suggests an expression cloning strategy for T-cell antigens. Proc Natl Acad Sci U S A. 1992;89(13):6020-6024.

50. Burgdorf S, Kautz A, Bohnert V, Knolle PA, Kurts C. Distinct pathways of antigen uptake and intracellular routing in CD4 and CD8 T cell activation. Science. 2007;316(5824):612-616.

51. Jancic C, et al. Rab27a regulates phagosomal $\mathrm{pH}$ and NADPH oxidase recruitment to dendritic cell phagosomes. Nat Cell Biol. 2007;9(4):367-378.

52. Kutomi G, et al. Targeting to static endosome is required for efficient cross-presentation of endoplasmic reticulum-resident oxygen-regulated protein 150-peptide complexes. J Immunol. 2009; 183(9):5861-5869.

53. Smerdou C, Liljestrom P. Two-helper RNA system for production of recombinant Semliki forest virus particles. J Virol. 1999;73(2):1092-1098.

54. Segura E, Villadangos JA. Antigen presentation by dendritic cells in vivo. Curr Opin Immunol. 2009;21(1):105-110.

55. Helft J, Ginhoux F, Bogunovic M, Merad M. Origin and functional heterogeneity of non-lymphoid tissue dendritic cells in mice. Immunol Rev. 2010;234(1):55-75

56. Asano K, et al. CD169-positive macrophages dominate antitumor immunity by crosspresenting dead cell-associated antigens. Immunity. 2011; 34(1):85-95.

57. Yamasaki S, Ishikawa E, Sakuma M, Hara H, Ogata $\mathrm{K}$, Saito T. Mincle is an ITAM-coupled activating receptor that senses damaged cells. Nat Immunol. 2008;9(10):1179-1188.

58. Joffre OP, Sancho D, Zelenay S, Keller AM, Reis e Sousa C. Efficient and versatile manipulation of the peripheral CD4+ T-cell compartment by antigen targeting to DNGR-1/CLEC9A. EurJ Immunol. 2010;40(5):1255-1265.
59. Goodridge HS, et al. Differential use of CARD9 by dectin- 1 in macrophages and dendritic cells. J Immunol. 2009;182(2):1146-1154.

60. Belizaire R, Unanue ER. Targeting proteins to distinct subcellular compartments reveals unique requirements for MHC class I and II presentation. Proc Natl Acad Sci US A. 2009;106(41):17463-17468.

61. Savina A, et al. NOX2 controls phagosomal $\mathrm{pH}$ to regulate antigen processing during crosspresentation by dendritic cells. Cell. 2006;126(1):205-218.

62. Accapezzato D, et al. Chloroquine enhances human $\mathrm{CD} 8+\mathrm{T}$ cell responses against soluble antigens in vivo. J Exp Med. 2005;202(6):817-828.

63. Delamarre L, Couture R, Mellman I, Trombetta ES. Enhancing immunogenicity by limiting susceptibility to lysosomal proteolysis. J Exp Med. 2006;203(9):2049-2055.

64. Delamarre L, Pack M, Chang H, Mellman I, Trombetta ES. Differential lysosomal proteolysis in antigen-presenting cells determines antigen fate. Science. 2005;307(5715):1630-1634.

65. Cebrian I, et al. Sec22b regulates phagosomal maturation and antigen crosspresentation by dendritic cells. Cell. 2011;147(6):1355-1368.

66. Zehner M, et al. Mannose receptor polyubiquitination regulates endosomal recruitment of $\mathrm{p} 97$ and cytosolic antigen translocation for cross-presentation. Proc Natl Acad Sci US A. 2011;108(24):9933-9938.

67. Ahrens $\mathrm{S}$, et al. F-actin is an evolutionarily-conserved damage-associated pattern recognized by DNGR-1, a receptor for dead cells. Immunity. In press.

68. Apetoh L, et al. Toll-like receptor 4-dependent contribution of the immune system to anticancer chemotherapy and radiotherapy. Nat Med. 2007;13(9):1050-1059.

69. Ginhoux F, et al. The origin and development of nonlymphoid tissue CD103+ DCs. J Exp Med. 2009;206(13):3115-3130.

70. GeurtsvanKessel CH, et al. Clearance of influenza virus from the lung depends on migratory langerin + CD11b- but not plasmacytoid dendritic cells. J Exp Med. 2008;205(7):1621-1634.

71. Reading PC, Whitney PG, Barr DP, Smyth MJ, Brooks AG. NK cells contribute to the early clearance of HSV-1 from the lung but cannot control replication in the central nervous system following intranasal infection. Eur Immunol. 2006;36(4):897-905.

72. Iborra S, Izquierdo HM, Martínez-López M, Blanco-Menéndez N, Reis e Sousa C, Sancho D. The DC receptor DNRG1 mediates cross-priming of CTLs during vaccinia virus infection in mice.J Clin Invest. 2012;122(5):1628-1643.

73. Inaba K, et al. Generation of large numbers of dendritic cells from mouse bone marrow cultures supplemented with granulocyte/macrophage colony-stimulating factor. J Exp Med. 1992;176(6):1693-1702.

74. Kappler J, White J, Wegmann D, Mustain E, Marrack P. Antigen presentation by Ia+ B cell hybridomas to H-2-restricted T cell hybridomas. Proc Natl Acad Sci U S A. 1982;79(11):3604-3607. 